International Journal of Physical Modelling in Geotechnics

Volume 15 Issue 4

Development of a real-time hybrid testing method in a centrifuge Kong, Cassidy and Gaudin
International Journal of Physical Modelling in Geotechnics,

2015, 15(4), 169-190

http://dx.doi.org/10.1680/ijpmg.14.00021

Paper 1400021

Received 17/07/2014 Accepted 06/01/2015

Published online 06/08/2015

Keywords: centrifuge modelling/offshore engineering/

soil-structure interaction

ICE Publishing: All rights reserved ice

$\overline{\text { Institution of Civil Engineers }}$

\title{
publishing
}

\section{Development of a real-time hybrid testing method in a centrifuge}

1 Vickie Kong BEng, MEng, PhD, MICE

Senior Engineer, Advanced Geomechanics, Nedlands, WA Australia

2 Mark Jason Cassidy BE(Hon), DPhil, FIEAust, FTSE ARC Laureate Fellow and Lloyd's Register Foundation Chair, Centre for Offshore Foundation Systems and ARC CoE for Geotechnical Science and Engineering, University of Western Australia, Crawley, Perth, WA, Australia
3 Christophe Gaudin MSC, MEng, PhD

Professorial Fellow, Centre for Offshore Foundation Systems and ARC CoE for Geotechnical Science and Engineering, University of Western Australia, Crawley, Perth, WA, Australia
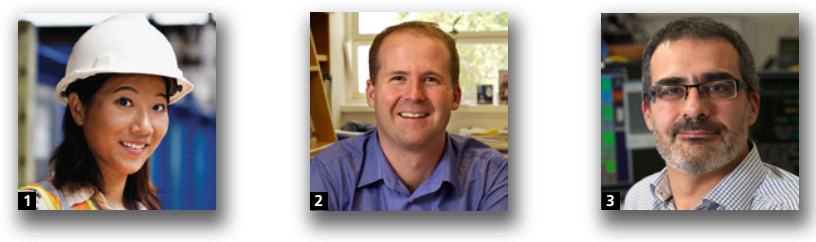

This paper presents a pioneering real-time hybrid testing method for geotechnical centrifuges. The method was used to investigate the behaviour of a jack-up leg reinstalled near an existing footprint, a problem that is highly nonlinear, stress dependent and involves complex soil-structure interactions. By physically modelling only the footprintleg interaction and numerically modelling the rest of the jack-up structure, the method enables a realistic account of all the parameters involved in the interaction, including the footprint geometry, soil heterogeneity and structural properties of the jack-up unit. The paper also details the three-degree-of-freedom actuators developed to model the interaction, which features controlled loads and motions along the vertical, horizontal and moment directions, and the real-time algorithm that bridges the physical and numerical models. The algorithm allows the stiffness on each axis to be varied. Testing performed at $1 \mathrm{~g}$ and in the centrifuge, modelling jack-ups with different stiffness on the horizontal and moment axes, is presented to validate the apparatus and methodology. The hybrid apparatus and real-time testing method were found to produce much more realistic boundary constraints than previous fixedsystem apparatuses, and this allowed the test results to be considerably more informative, accurate and useful.

\section{Introduction}

Real-time hybrid testing has seen increased popularity in testing complex structural systems but has seen very limited application in geotechnics. In real-time hybrid testing, a physical model of a critical element is combined with a numerical model of the remainder of the system. In this way, an accurate test on the element of interest can be physically established but with integrated behaviour of the entire system still accounted for.

Typically, actuators apply displacements to a physical model, and the physical test interacts with a numerical model in real time by means of a feedback control loop. In this way, any rate-dependent components within the system are tested. The loop starts by applying external excitation to the numerical model. The response at the common point $(\mathrm{CP})$ between the physical model and the numerical model is evaluated using a time-stepping scheme. The displacements at the CP are imposed on the physical model by the actuator in real time (i.e. over the same period of time that the numerical model is stepping). The restoring forces are measured directly from the physical model at the end of the time step. These forces are fed back and imposed on the $\mathrm{CP}$ of the numerical model. The response of the numerical model to external load and feedback forces is evaluated for the end of the next time step. Looping continues until the external excitation ceases.

Early developments in hybrid testing were not in real time but used at an expanded time scale. For instance, in the testing undertaken by Hakuno et al. (1969), an electromagnetic actuator was used to load the physical model and an analogue 
computer analysed the numerical model. These were not fast enough to model the seismic response of a structure in real time. It was not until the introduction of digital computers and more powerful actuators that hybrid testing that interacted on a common time axis was first achieved by Nakashima et al. (1992). Recent advancements in real-time hybrid testing have concentrated on understanding experimental error, improving delay compensation algorithms and substructuring techniques. However, applications of the method remain in modelling of the dynamic behaviour of complex structural and mechanical systems, such as bridges, buildings and aircraft (Ayari, 2008; Blakeborough et al., 2001; Van der Auweraer et al., 2008; Wagg et al., 2008).

The use of hybrid testing within geotechnics remains rare. The first application of hybrid testing in geotechnical engineering occurred in 1975, wherein the dynamic performance of a pile foundation was investigated (Mochizuki, 1975). Subsequent development concentrated on evaluating the potential for one-dimensional liquefaction. In such studies, the liquefiable layer was physically modelled by a soil element test, whereas a numerical model was applied to the remaining layers (Adachi et al., 1998; Fujii et al., 2000; Katada and Hakuno, 1982; Kusakabe and Morio, 1995; Sento et al., 2002). In these studies, the soil deformation caused by liquefaction both during and after an earthquake was evaluated. Various researchers have concluded that hybrid testing provides an accurate prediction of the dynamic soil response without the use of idealised constitutive relations (e.g. Kazama et al., 2004; Kwon et al., 2007; Takahashi et al., 2003).

The application of hybrid testing to the study of soil-structure interaction is even more limited. Five studies are known to the authors. Mochizuki (1975) physically tested the soil and the pile, with the rest of the building structure modelled numerically as lumped masses. Kobayashi et al. (2002) also physically modelled the pier and pile foundation in the laminar box of a shake table, with the rest of the bridge structure modelled numerically. In other studies, it was actually the soil and foundation system that were modelled numerically by means of a finite element or lumped mass model (Ohtomo et al., 2008; Spencer et al., 2006; Wang et al., 2009). To the authors' knowledge, there has been no use of real-time hybrid testing within a geotechnical centrifuge.

The purpose of this paper is to describe the underlying principles and the design criteria required to apply real-time hybrid testing within a geotechnical centrifuge. These principles and criteria were used to design a new vertical-horizontal rotation actuator system (named the VHM actuator) to be used within a beam centrifuge to investigate the behaviour of a jack-up platform foundation during penetration nearby a footprint. Details of its design, validation and application are provided. The control of the hybrid tests was found to be critical and the real-time control system developed is presented in detail. Results of experimental validation of the system at both $1 \mathrm{~g}$ and in the centrifuge are provided to illustrate the effectiveness of the real-time hybrid testing methodology.

\section{Motivation}

The real-time hybrid testing method has been developed to test the reinstallation of a mobile jack-up platform next to existing seabed footprints.

Jack-ups are self-elevating mobile units operating in offshore oil and gas fields that are situated in water depths of up to approximately $120 \mathrm{~m}$. A typical jack-up unit consists of a floatable hull platform and three independent retractable trusswork legs, each resting on a spudcan footing. The spudcans are typically between 10 and $20 \mathrm{~m}$ in diameter (e.g. Menzies and Roper, 2008; Zhang et al., 2011). During the removal of a jack-up unit from a site, the extraction of spudcans leaves significant seabed depressions. In the offshore industry, these are referred to as footprints. Jack-up units often have to return to the same site and be installed near or into existing footprints (Figure 1). This is a problematic operation because the spudcan located near the footprint is subjected to eccentric and/or inclined loading conditions. This can lead to structural failures within the jack-up legs. Furthermore, movements can occur in the spudcan and jack-up legs, with potential risks that the jack-up hull may hit the nearby fixed platform.

The physical testing of this reinstallation process is extremely complex, with interacting features of soil and structural mechanics. The system response of the jack-up unit could be studied experimentally by modelling a full three-legged jack-up model, as was adopted to measure the combined loading of a jack-up subjected to pushover (storm) forces in a centrifuge by Dean et al. (1996) and Bienen et al. (2006, 2009), and on the laboratory floor by Vlahos et al. $(2005,2008,2011)$ and Cassidy et al. (2010). However, in these cases, the jack-up remained in one configuration with the entire model initially loaded vertically (installation) and then laterally (storm pushover). Installation into a footprint is more complex, with usually only one spudcan installed at a time. One common industrial practice is to orient the jack-up unit so that only one leg is located near the footprint (Figure 1). The other two legs are installed first to increase the rigidity of the system and help restrain movement. Moving legs of a full three-legged jack-up unit independently is extremely difficult to model physically, particularly to automate at high gravitational levels in the confined space of a geotechnical centrifuge. Furthermore, even if it was possible, a full three-legged jack-up model requires a relatively large testing space and restricts the modelling to one structural configuration (both limiting the number and scope of any parametric study). 

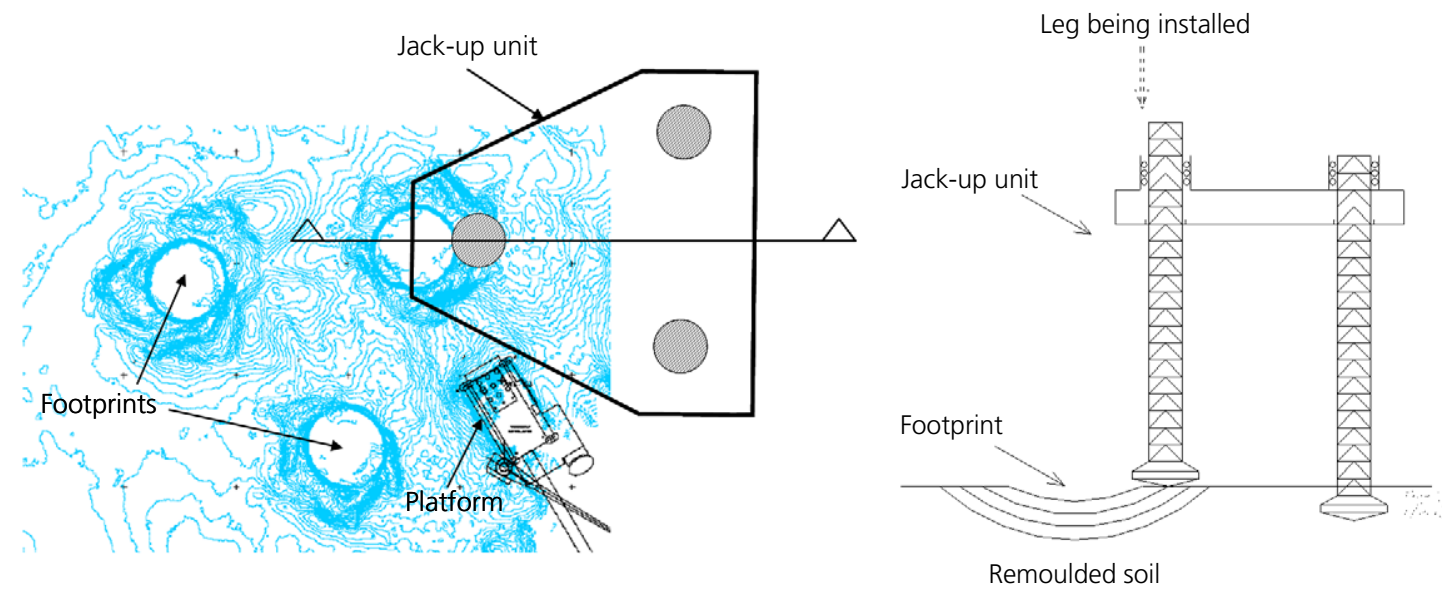

Figure 1. Scenario of one jack-up leg reinstalled near a footprint (contour plot of footprint after Gan, 2009)

Due to these difficulties, all of the existing testing of the reinstallation of jack-ups next to footprints has been limited to testing of a single spudcan and leg. The effect of the surrounding jack-up structure has been effectively ignored. Conditions have been either fully fixed, with a rigid connection between the model leg and the vertically displacing actuator (Cassidy et al., 2009; Gan et al., 2012; Kong et al., 2013; Stewart and Finnie, 2001), or fixed vertically and rotational but free-to-slide laterally, with a roller connection adopted in the horizontal direction (Gaudin et al., 2007). As the former assumption ignores the finite structural flexibility of the jack-up unit, it precludes any measurement of jack-up movement during reinstallation tests and the measured loads therefore represent an overestimation of typical behaviour. On the other hand, the slider has no lateral flexibility (say from the other two pinned in legs, Figure 1) and is over-estimating movements and underestimating loads. The use of a mechanical connection piece to represent the leg-hull connection and overall jack-up structure is also limited as the design for multiple degrees of freedom is complex and it is not mechanically feasible to change the stiffness due to the non-linear behaviour during reinstallation.

\section{Real-time hybrid testing of jack-up platform reinstallation}

The soil-structure interaction associated with the reinstallation of a jack-up leg near a footprint is a complex problem that can be simplified by using real-time hybrid testing. The critical component is the interaction of the leg/spudcan with the footprint. The interaction is highly non-linear and time dependent and is difficult to model numerically. Consequently, real-time testing was used so that the rate-dependent response and drainage conditions of the soil were correctly modelled and were consistent with the jack-up installation speed used offshore.
The remainder of the jack-up unit was numerically modelled and described by a load-displacement relationship. A real-time control algorithm was constructed so that the physical model interacted with the numerical model at a CP located along the jack-up leg, as illustrated in Figure 2. The test was initiated with a pure vertical displacement of the $\mathrm{CP}$ (simulating the start of the leg installation). Following that, and in each subsequent loop, the loading (combining vertical $V$, horizontal $H$ and moment $M$ loads as described later in the paper) at the $\mathrm{CP}$ was derived through measurements in the physical jack-up model. These measurements were inputted to the numerical model, which in turn calculated the vertical and horizontal displacements and rotations ( $v, h$ and $\theta$ ) to be imposed on the physical jack-up leg during the next step.

\section{Physical model of the jack-up unit}

\subsection{Jack-up model}

The physical model consists of a model jack-up leg and spudcan foundation. A prototype jack-up unit that was $94 \mathrm{~m}$ tall was considered, the properties of which are shown in Table 1 and Figure 3. The CP that defined the junction between the physical and the numerical model was located at $23.5 \mathrm{~m}$ above the spudcan ( $235 \mathrm{~mm}$ in model dimensions). The spudcan was modelled as a flat-base footing $6 \mathrm{~m}$ in diameter and $0.7 \mathrm{~m}$ deep (solid aluminium model of $60 \mathrm{~mm}$ diameter and $7 \mathrm{~mm}$ thick). The flat-base footings were used to simplify the experiments by eliminating the variables related to the spudcan geometry, including the apex angle, central spigot and cone angle. The model leg and spudcan are shown in Figure 4.

A lower leg flexural rigidity (EI) than observed in field jack-ups was purposely used. This is because the diameter of 


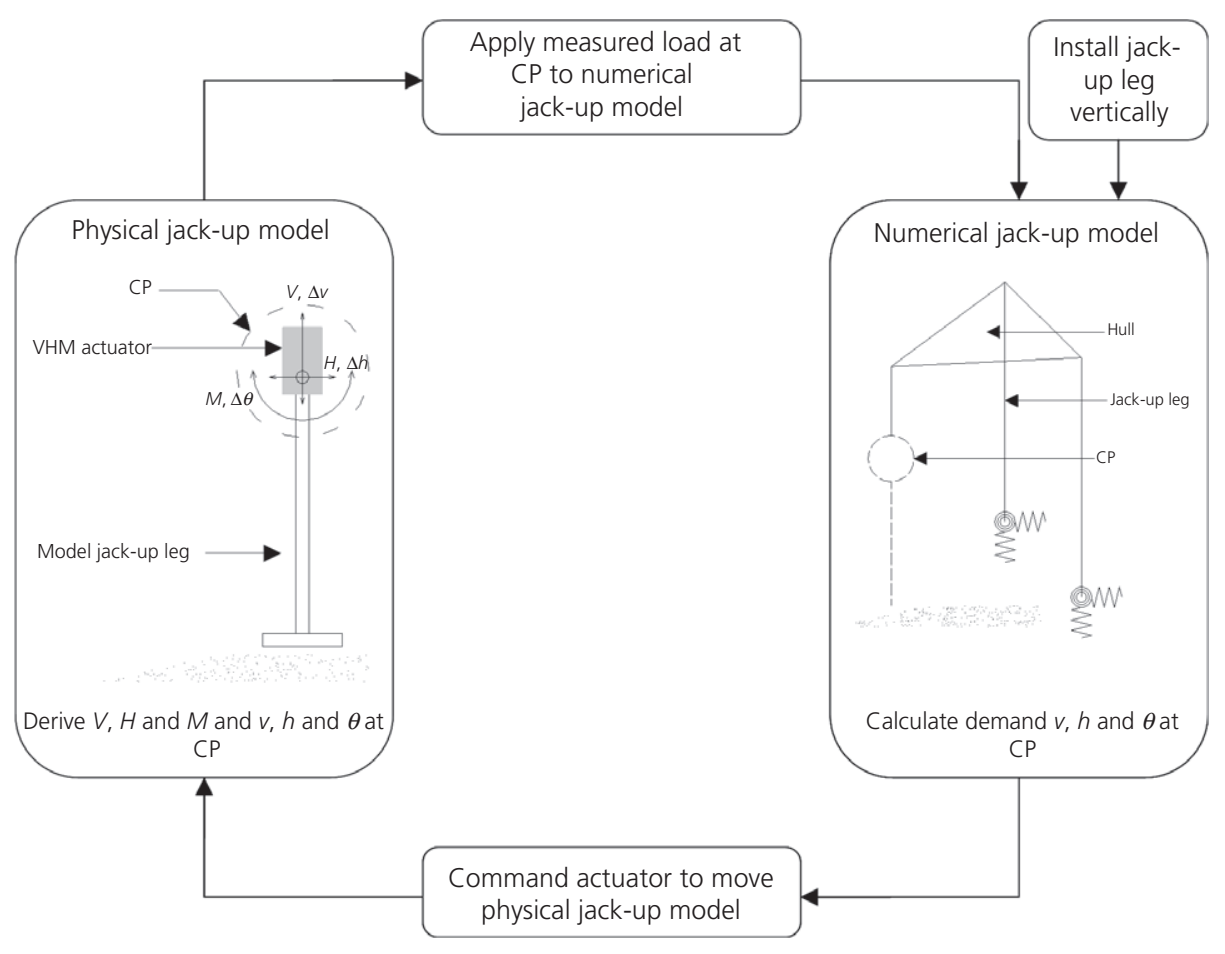

Figure 2. Concept of real-time hybrid testing in jack-up

reinstallation near an existing footprint

\begin{tabular}{|c|c|c|c|}
\hline Dimension & Model & $\begin{array}{l}\text { Scaling } \\
\text { factor }\end{array}$ & Prototype \\
\hline Leg length, $L$ & $940 \mathrm{~mm}$ & $N$ & $94 \mathrm{~m}$ \\
\hline Leg spacing, $W$ & $600 \mathrm{~mm}$ & $N$ & $60 \mathrm{~m}$ \\
\hline Height to $C P$ & $235 \mathrm{~mm}$ & $N$ & $23 \cdot 5 \mathrm{~m}$ \\
\hline $\begin{array}{l}\text { Second moment of } \\
\text { inertia of leg, I }\end{array}$ & $6 \cdot 5 \times 10^{-10} \mathrm{~m}^{4}$ & $N^{4}$ & $0.065 \mathrm{~m}^{4}$ \\
\hline $\begin{array}{l}\text { Cross-sectional area } \\
\text { of leg, } A\end{array}$ & $2.5 \times 10^{-5} \mathrm{~m}^{2}$ & $N^{2}$ & $0.25 \mathrm{~m}^{2}$ \\
\hline Diameter of spudcan, $D$ & $60 \mathrm{~mm}$ & $N$ & $6 \mathrm{~m}$ \\
\hline
\end{tabular}

For the scaled model at $1 \boldsymbol{g}$, a 1:100 numerical model was established

Table 1. Model and prototype dimensions of jack-up unit models

the spudcan was also smaller. With the consideration on the maximum number of test to be conducted within a soil sample and the correctness of scaled spudcan size, the diameter of spudcan adopted in this model was $6 \mathrm{~m}$, which is around three times smaller than the typical dimension of a spudcan used in practice. The moment acting on the testing spudcan would therefore be 27 times smaller than on an 18 m spudcan (with moment proportional to the cubic of footing diameter). Since the main purpose of this study was to investigate the loaddisplacement responses during jack-up reinstallation, the leg flexural rigidity was also scaled down proportionally to maintain the bending properties. The leg flexural rigidity adopted was of a similar reduced ratio, with $\mathrm{EI}=1.3 \times 10^{10} \mathrm{~N} \mathrm{~m}^{2}$, approximately 5-100 times lower than the range of values reported in other publications (e.g. $\mathrm{EI}=7.12 \times 10^{10} \mathrm{~N} \mathrm{~m}^{2}$ and $\mathrm{EI}=$ $1.44 \times 10^{12} \mathrm{~N} \mathrm{~m}^{2}$ in Murff et al. (1991) and Vlahos (2004), respectively).

During penetration near an existing footprint, the jack-up leg experiences combined vertical $V$, horizontal $H$ and moment $M$ loads and motions. To accommodate and monitor these loads and motions, the jack-up leg is connected to a purposely designed VHM actuator, and instrumented with axial gauges and bending gauges, the readings of which are converted into loads $H_{\mathrm{CP}} \quad V_{\mathrm{CP}}$ and $M_{\mathrm{CP}}$ at the $\mathrm{CP}$ (left-hand side of Figure 2). The sign conventions and definitions of terminologies adopted in the control algorithm, as also relative to the footprint, are presented in Figure 4. A positive horizontal force at the load reference point (LRP) indicates that the footing was resisting a movement towards the footprint and a positive moment indicates that the footing was resisting a counter-clockwise rotation away from the footprint. 

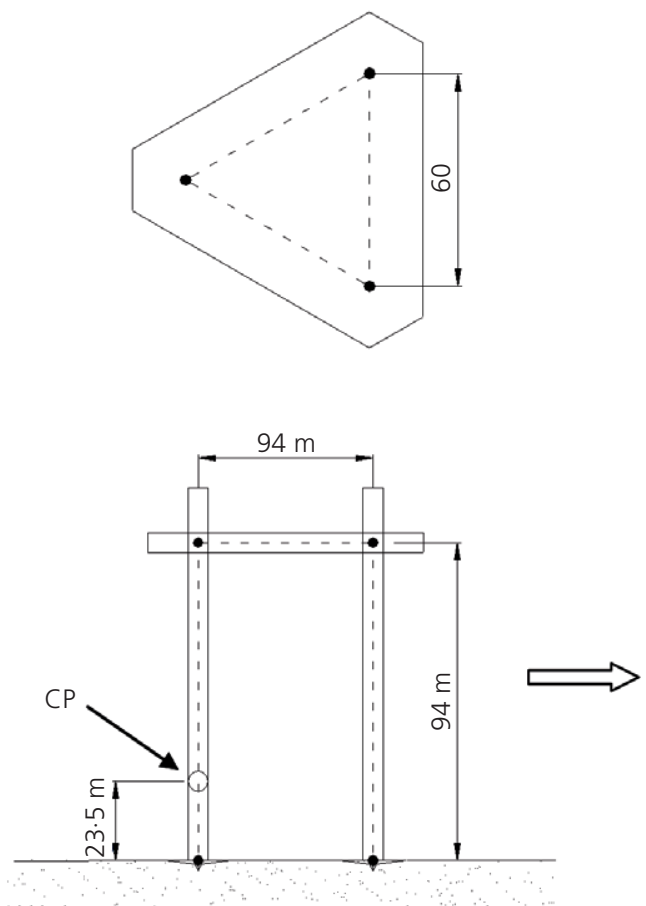

Jack-up properties:

Young's modulus $E \quad 200 \mathrm{GPa}$

Shear modulus $G \quad 8000 \mathrm{MPa}$

Poisson ratio $\quad 0.25$

Leg equivalent I $\quad 0.065 \mathrm{~m}^{4}$

Leg equivalent A $\quad 0.250 \mathrm{~m}^{2}$

Hull equivalent I $\quad 10 x \operatorname{leg}$ I

Hull equivalent $A \quad 50 x \operatorname{leg} A$

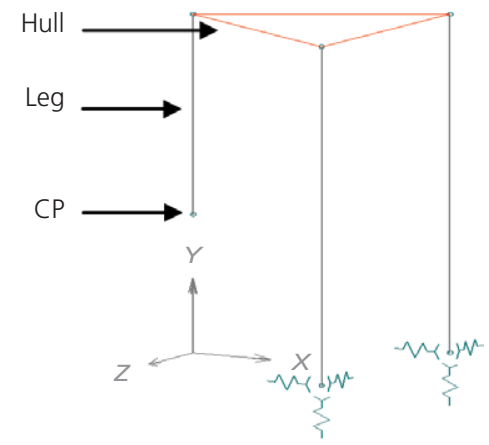

Figure 3. Numerical model of the simplified jack-up unit assumed in the experiments

\subsection{Design of the VHM actuator}

An actuator with three degrees of freedom was required to allow the jack-up model leg and spudcan to move independently in the vertical, horizontal and rotational direction. As the existing two-axis linear actuator at University of Western Australia (UWA) only provides horizontal and vertical movement, an additional angular actuator that can be attached to the existing linear actuator was developed.

The limited space in the existing linear actuator unit precluded the use of a pair of actuators to generate the angular movement (such as used in the design of three-degrees-of-freedom VHM actuators for beam centrifuge by Dean et al. (1997) and Punrattansin et al. (2003) and more recently in the UWA drum by Zhang et al. (2013, 2014a, 2014b, 2014c)). Therefore, an innovative pulley system was developed for use in this study. As shown in Figures 5 and 6, the rotational motion of the motor was converted to angular motion at a pivot point through a pulley system. The main advantage of this system is that there is only one pivot point in the system and the single motor module is aligned with the line of action. This generates precise angular movement from a relatively slim configuration.

Figure 7 shows the angular actuator's key components: the motor module, the cable and pulley, the pivot point and the actuator box. The new angular actuator has outer dimensions of $100 \mathrm{~mm} \times 79 \mathrm{~mm} \times 411 \mathrm{~mm}$ (width $\times$ thickness $\times$ height $)$ and a weight of $2.57 \mathrm{~kg}$. After accommodating the angular actuator, the stroke in the linear actuators was reduced to $180 \mathrm{~mm}$ (vertical) and $70 \mathrm{~mm}$ (horizontal). A detailed discussion of the design and fabrication of the key components is presented in Appendix 1, as well as in Kong (2012).

Table 2 shows the specification of the VHM actuator. The angular actuator was designed to have a maximum angular stroke of $3^{\circ}$ in both the clockwise and anti-clockwise directions. This limit was set because tilting of a jack-up unit to more than $2^{\circ}$ at the platform level would not normally be allowed for offshore (due to possible damage of the leg-hull connection, as advised by Purwana, Personal Communication (2010)). In this study, it was conservatively assumed that all tilting at the platform level resulted from angular movement of the jack-up leg installed near the footprint.

Another major advantage of the VHM actuator is that its centre of rotation can be prescribed anywhere along the vertical axis. When the angular actuator rotates around the pivot point $(\Delta \theta)$, the linear actuators can also move the pivot point in vertical and horizontal directions ( $\Delta x$ and $\Delta y)$, offsetting the linear movement so that there is pure rotational movement at any prescribed point along the vertical axis. 


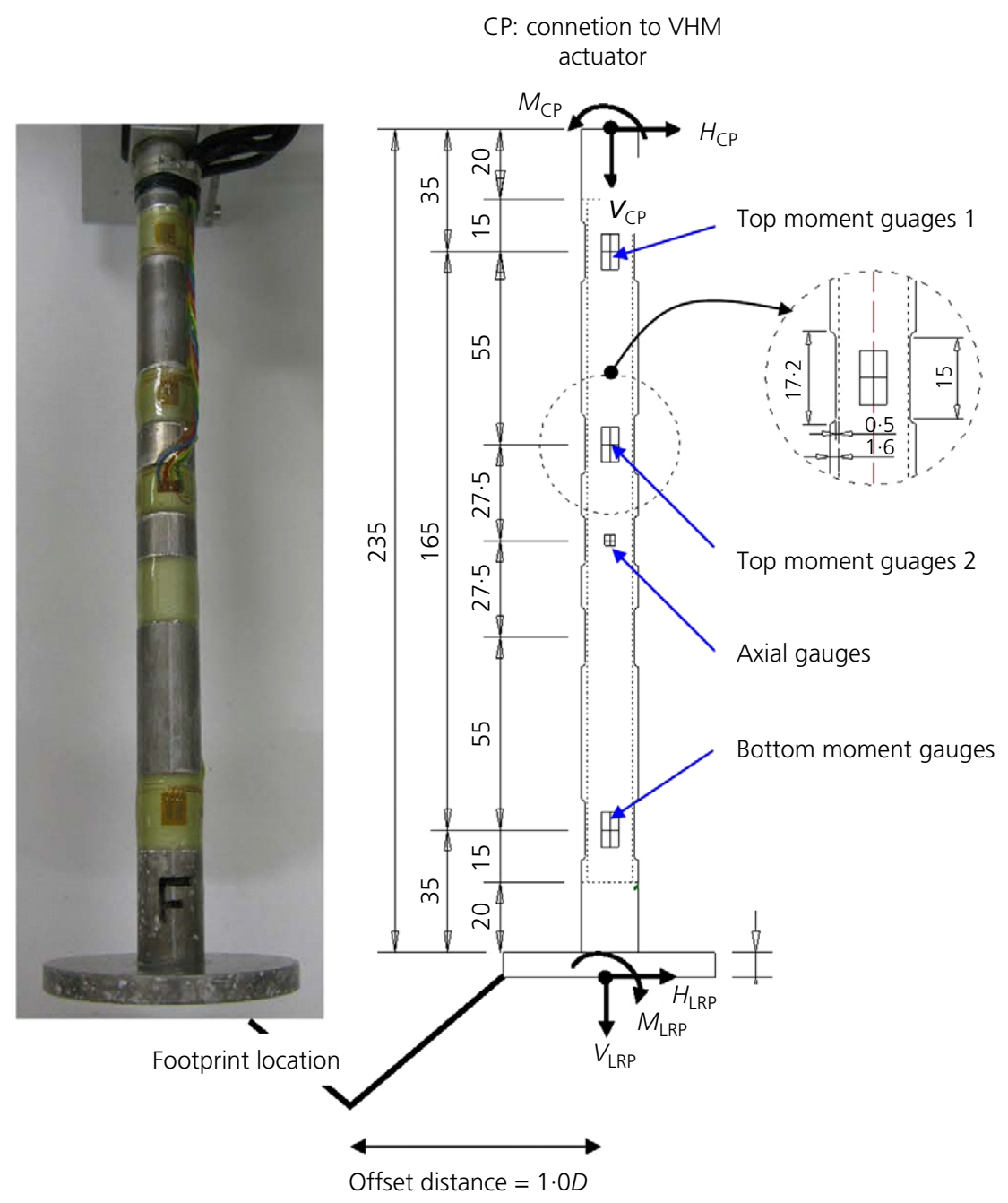

Figure 4. Model jack-up leg and footing used in real-time hybrid testing (all units in $\mathrm{mm}$ )

\subsection{Numerical model}

While the section of the jack-up leg located near the footprint was modelled physically, the remainder of the jack-up unit was simplified and modelled numerically (right-hand side of Figure 2). The jack-up leg and the hull were modelled as beam elements, and the leg-hull connection was simplified and modelled as a fully fixed node. The two pre-embedded footings were modelled as a series of linear springs, with stiffness determined according to the Boussinesq elastic solutions set out in the Society of Naval Architects and Marine Engineers (SNAME) industry guidelines (SNAME, 2002). The soil properties and foundation stiffness adopted in the analyses are shown in Table 3.

To reduce the complexity of the initial testing programme, the numerical model was assumed to be linear elastic. The numerical model could therefore be reduced to a simple flexibility (inverse stiffness) matrix at the CP. The governing load-displacement relationship in the general form is presented in Figure 8. The matrix was evaluated by simply applying unit forces at the CP into the structural model, with the resultant displacement and angular movement at the $\mathrm{CP}$ divided by the unit load to determine the flexibility. Structural analysis program SPACEGASS was used for this numerical calculation (Integrated Technical Software Pty Ltd, 2010). Further details, including example calculations, are available in Kong (2012). Jack-up units with different flexibilities could therefore be easily modelled by introducing different flexibility matrices of the following form

1. $\left(\begin{array}{c}\Delta h \\ \Delta v \\ \Delta \theta\end{array}\right)=\left(\begin{array}{lll}C_{H h} & 0 & C_{M h} \\ C_{H v} & 0 & C_{M h} \\ C_{H \theta} & 0 & C_{M h}\end{array}\right) \times\left(\begin{array}{c}\Delta H_{\mathrm{cp}} \\ 0 \\ \Delta M_{\mathrm{cp}}\end{array}\right)+\left(\begin{array}{c}0 \\ \Delta v_{\text {step }} \\ 0\end{array}\right)$ 
International Journal of Physical Modelling in Geotechnics Volume 15 Issue 4
Development of a real-time hybrid

testing method in a centrifuge

Kong, Cassidy and Gaudin

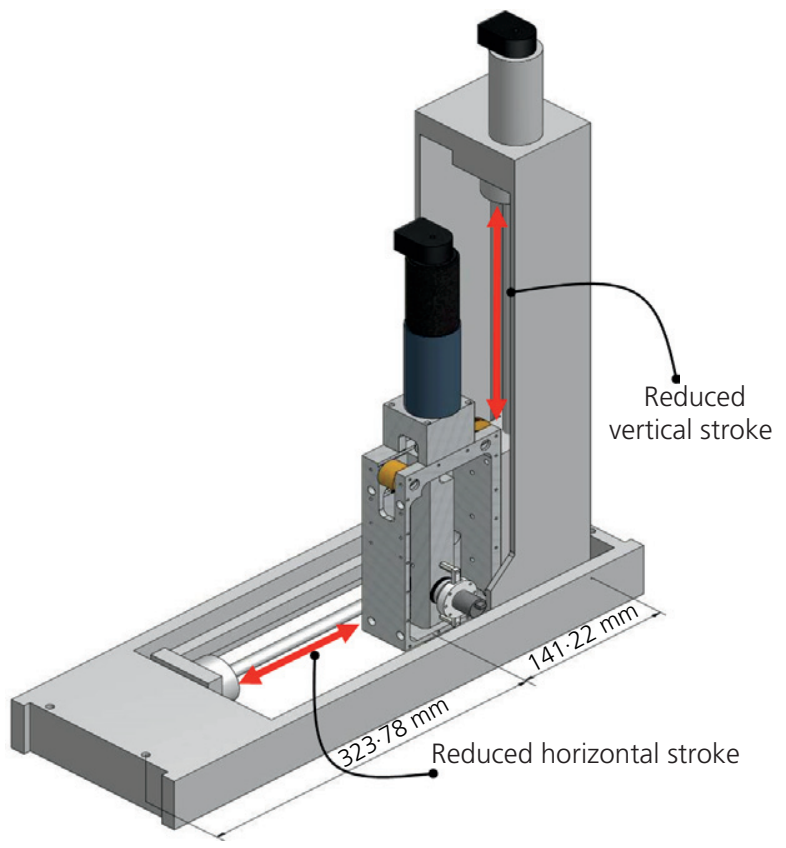

Figure 5. The two-axis linear actuator with the additional rotational unit

The following assumptions were made to further simplify the problem

- the vertical load-displacement cross-coupling terms $C_{V h}$, $C_{V v}$ and $C_{V \theta}$ were eliminated, and the installation of the jack-up leg was modelled by the application of a constant vertical displacement step $\Delta v_{\text {step }}$

- the current real-time hybrid testing method considered constant jack-up flexibility throughout reinstallation, so the non-linear jack-up load-displacement responses due to the $P-\Delta$ term were ignored

- in theory, as the physical model leg penetrates into the soil, the length of the jack-up leg in the numerical model must increase by the same length. This change in jack-up leg length (and therefore jack-up flexibility) was not considered in the numerical model.

These assumptions were necessary for the development of a relatively simple control algorithm. However, the test results still provided fundamental information regarding the effect of the jack-up's structural properties on the reinstallation response. However, the prototype being simulated still differs from an offshore installation, as the numerical model did not include any plasticity in the spudcan response, any redistribution of loads, including due to a change of the jack-up's centre of gravity, and structural dynamics effects were ignored. However, the current control algorithm could be modified to update jack-up flexibility in real time in a future study. This would require a dynamic structural analysis code to update continually the flexibility at the CP.

\subsection{Real-time control algorithm}

In the real-time hybrid test, the VHM actuator applied displacements $v, h$ and $\theta$ to the physical jack-up leg model, and the physical test interacted with a numerical model in real time by means of a feedback control loop, allowing the complete jack-up unit to be tested. Figure 9 outlines the system and the communication of forces and displacements to the hardware, which controls the flow loop outlined in Figure 2 and allows the hybrid tests to be run. The control loop had the following features

the loads acting on the physical jack-up model were measured by strain gauges (Figure 4)

- the load readings were transferred to the DigiDAQ Box, which is an analogue-to-digital converter system (Gaudin et al., 2009)

- the load readings were then transferred to the flight computer mounted on the central platform of the centrifuge (flight PC) through an ethernet connection

- in the flight PC, the load reading was streamed to two programmes

the data logging system DigiDAQ

- the real-time control algorithm and numerical model programme

- the numerical model programme computed the demand displacement and angular movement (demand)

- the demand was fed into DigiDAQ for logging, transferred to the modified main actuator control software PACS (packaged actuator control system; refer to De Catania et al. (2010) for details) and subsequently transferred to the Servo Controller (National Instrument NI7344-PCI)

- the demand (in the form of an analogue signal) was amplified through a linear power amplifier

- the VHM actuator moved with an inner control loop to achieve accurately the demands $v, h$ and $\theta$.

The numerical jack-up model was written in LabVIEW and incorporated into the real-time control loop, minimising any potential decay in communication. The flight computer is continuously connected to the remote control PC in the control room. High-speed communication between the two PCs is achieved with optical fibre cables and slip rings, which are housed within the main axis of the beam centrifuge. The test was controlled using the remote control PC, and the data were displayed in real time for monitoring. The maximum operating frequency of this control system is $100 \mathrm{~Hz}$.

The control loop starts by applying a constant vertical displacement $\Delta v_{\text {step }}$, simulating the installation of the jack-up leg at a 


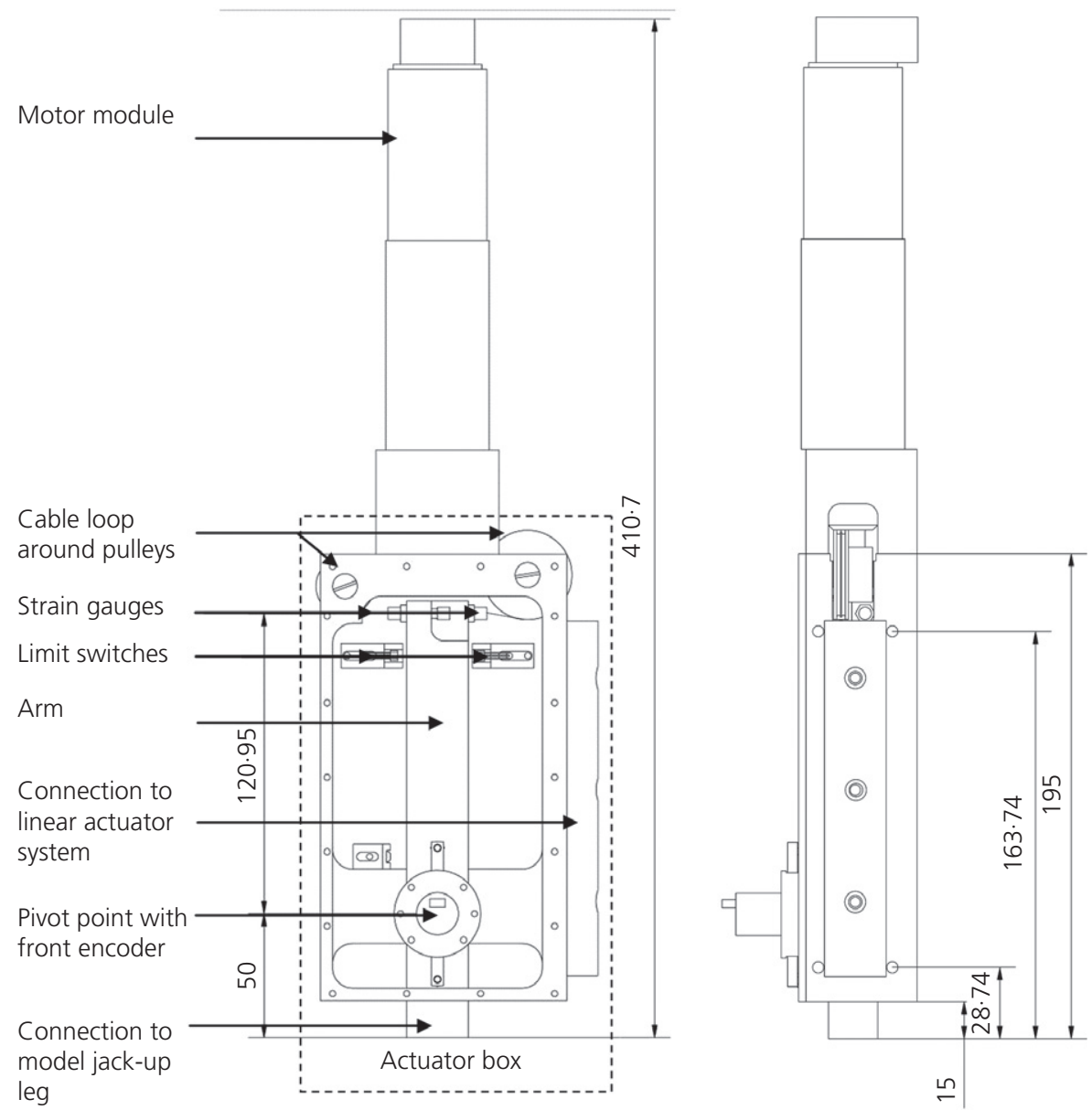

Figure 6. Details of the additional rotational unit (dimensions in $\mathrm{mm}$ )

constant vertical rate (step 1 in Figure 10). The loads acting on the physical jack-up model are measured by the strain gauges $\left(\mathrm{VHM}_{i}\right.$ in step 2) and fed into the numerical model. The measured load is first converted to loads at the CP between the physical model and the numerical model using a timestepping scheme $\left(\mathrm{VHM}_{\mathrm{CP} i}\right.$ in step 3$)$. The block average value, $\mathrm{VHM}_{\text {ave } i}$, is evaluated to filter the noise in the measured data (step 4). This block average is then compared with the previous measured maximum load to determine the location of the physical jack-up leg during a loading or unloading condition (steps 5, 6a, 6b). The demand displacement and angular movement are determined by solving the load-displacement relationship (step 7), and the demands are then imposed on the physical model by the actuator in real time (i.e. over the same period of time that the numerical model is stepping). At the end of the time step, the loads and the actuator position are measured and fed back into the numerical model, thus closing the loop (step 9a). This loop is repeated until the physical model jack-up leg reaches the designated maximum penetration depth (step 9b).

The load readings from the bending gauges introduced considerable high-frequency noise content to the signal being fed back into the numerical model. With the load-displacement relationship, this high-frequency loading can result in highfrequency cyclic motion. This becomes of particular concern as the soil sample may be undesirably remoulded at relatively shallow penetration where loading is small and the movement was dominated by the noise. This problem, and a solution of mitigation by using a filtering system, has been reported by, among others, Wagg et al. (2008). In this study, the average loadings are evaluated from block of load readings of sample size $F$, where $F$ is defined here as the number of load reading per block. The demand displacement and angular movement are then calculated and implemented over the next block time step $f$, where $f$ is defined as the time between the first 


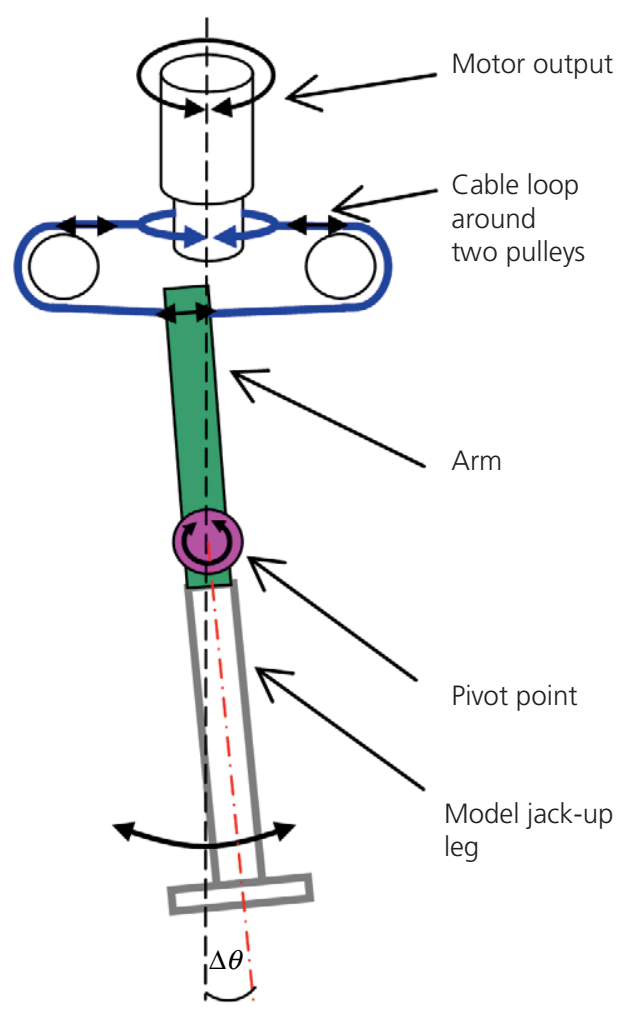

- Providing the rotational motion

- Cable tied around two pulleys, forming a loop

- Connecting to the motor output and an arm, forming a pulley system

- Converting rotational movement to translational movement

- Connecting the cable loop to the pivot point

- Converting translational movement to angular movement around the pivot point

- Providing a connection to attach the model jack-up leg at the end of arm

- Providing angular movement $(\theta)$

Figure 7. Key components of angular actuator's pulley system

and last readings of the block of load reading $(f=F-1)$. The high-frequency noise can effectively be filtered from the system.

There are two prime sources of delay in the system, one from the time-step delay in the feedback loop and the other from the inherent dynamics of the actuators. Effort has been made to reduce the second source of delay. The loading required to drive the scaled physical model is relatively small. Therefore, the fast response servo-motor actuator was adopted instead of the hydraulic actuator. The proportional-integral-derivative control (refer to Goodwin et al., 2000) of each VHM actuator was tuned according to National Instruments (2009) to minimise delay and generate as accurate a motion as possible. The filter system introduces additional delay into the loop. The load reading was stored until obtaining $F$ number of load readings to calculate the demand displacement and angular movement for the next block time step $f$. The block sample size $F$ was, therefore, carefully selected balancing the delay and the noise level in the system.

\begin{tabular}{llll}
\hline & Vertical & Horizontal & Angular \\
\hline Maximum $\boldsymbol{g}$ & $250 \boldsymbol{g}^{\mathrm{a}}$ & $250 \boldsymbol{g}^{\mathrm{a}}$ & $100 \boldsymbol{g}^{\mathrm{b}}$ \\
Maximum stroke & $180 \mathrm{~mm}$ & $70 \mathrm{~mm}$ & $\pm 3^{\circ}$ \\
Maximum speed & $3 \mathrm{~mm} / \mathrm{s}$ & $30 \mathrm{~mm} / \mathrm{s}$ & $3 \cdot 96 \% \mathrm{~s}$ \\
Maximum force/moment & $V_{\max }=6500 \mathrm{~N}$ & $H_{\max }=2000 \mathrm{~N}$ & $M_{\max }=180 \mathrm{~N} \mathrm{~m}$ at pivot point \\
Movement precision & $2 \cdot 84 \times 10^{-5} \mathrm{~mm} /$ count & $2 \cdot 84 \times 10^{-5} \mathrm{~mm} /$ count & $1 \cdot 49 \times 10^{-5} /$ count \\
\hline
\end{tabular}

aThe original two-axis linear actuator is designed to operate up to $250 \mathrm{~g}$

${ }^{b}$ The angular actuator is designed to operate up to $100 \mathrm{~g}$

Table 2. General specifications for the VHM actuator 
Development of a real-time hybrid testing method in a centrifuge

Kong, Cassidy and Gaudin

\begin{tabular}{lll} 
Parameters & $\begin{array}{l}1 \boldsymbol{g} \text { model } \\
\text { tests }\end{array}$ & $\begin{array}{l}100 \boldsymbol{g} \text { beam } \\
\text { centrifuge tests }\end{array}$ \\
\hline$S_{\mathrm{u}}$ at $z / D=0.25$ & $5.0 \mathrm{kPa}$ & $4.7 \mathrm{kPa}$ \\
OCR at $z / D=0.25$ & 3000 & 4.8 \\
$K_{\mathrm{v}}$ & $111.9 \mathrm{kN} / \mathrm{m}$ & $52583.3 \mathrm{kN} / \mathrm{m}$ \\
$K_{\mathrm{h}}$ & $86.3 \mathrm{kN} / \mathrm{m}$ & $40542.5 \mathrm{kN} / \mathrm{m}$ \\
$K_{\text {rot }}$ & $0.07 \mathrm{kN} \mathrm{m} / \mathrm{rad}$ & $351165.0 \mathrm{kN} \mathrm{m} / \mathrm{rad}$
\end{tabular}

(a) The spring stiffness of the embedded footings is defined by the vertical stiffness $\left(K_{\mathrm{v}}\right)$, the horizontal stiffness $\left(K_{\mathrm{h}}\right)$ and the rotational stiffness $\left(K_{\text {rot }}\right)$ and is based on Boussinesq elastic solutions. The depth factor, defined by $k_{\mathrm{dv}}, k_{\mathrm{dh}}$ and $k_{\mathrm{drot}}$, was applied according to SNAME (2002) to incorporate the footing embedment depth. The footing embedment depth ratio z/D (depth of footing over diameter of footing) was assumed to be 0.25 and no backflow was assumed. Therefore, values of $k_{\mathrm{dv}}=1.15, k_{\mathrm{dh}}=1.33$ and $k_{\mathrm{dro}}=1.28$ were used. This is a conservative assumption as the embedment depth of the two footings varies greatly depending on the site condition and the embedment depth can be up to $2 D$ in practice

(b) The shear modulus of the soil was estimated to be $G=I_{r} S_{u}=\left(600 / O C R^{0 \cdot 25}\right) S_{u}$, based on Cassidy et al. (2004) and the SNAME guidelines

(c) A Poisson ratio of 0.5 was assumed to represent undrained clays

(d) The $s_{u}$ at $z / D=0.25$ is the upper bound values estimated from the $t$-bar test results shown in Figure 11

(e) The sample consolidated at $\mathbf{1} \boldsymbol{g}$ was under a pressure of $300 \mathrm{kPa}$, but only preconsolidated at $47 \mathrm{kPa}$ before spinning in the centrifuge

Table 3. Soil properties and foundation stiffness values (for ' $F_{\text {SNAME' }}$ ')

\section{Examples of results}

A series of 16 tests were performed, 10 tests at $1 \boldsymbol{g}$ and six in the centrifuge. The $1 g$ tests used exactly the same set-up, but just without accelerating the centrifuge. All results are detailed in Kong (2012) and eight tests are presented hereafter as examples (with details in Table 4).

\subsection{Soil sample}

In both the $1 \mathrm{~g}$ and centrifuge tests, an over-consolidated soil profile was targeted as it represents a profile in which deeper footprints can be created (due to higher surface strengths in comparison to normally consolidated soil). Kaolin clay soil that is used extensively at UWA was adopted in this test (Acosta-Martínez, Personal Communication (2008); Stewart, 1992). The Kaolin was mixed with de-aired water to form a slurry with a water content of $120 \%$ and consolidated in a hydraulic press. The maximum consolidation pressure in the

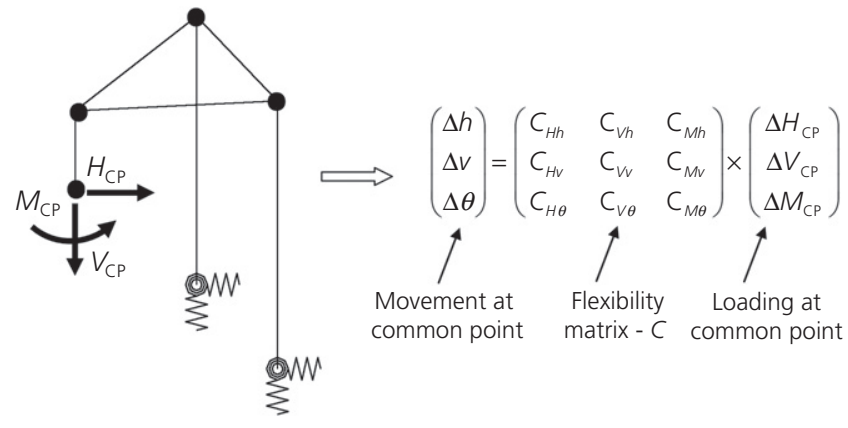

Figure 8. Linear-elastic numerical model of a jack-up unit

press was $300 \mathrm{kPa}$ for the $1 \mathrm{~g}$ tests and $47 \mathrm{kPa}$ for the centrifuge samples. After removal from the consolidation press, the soil sample was left to swell for $7 \mathrm{~d}$ before testing at $1 \mathrm{~g}$. In the centrifuge, the sample was immediately spun at $120 \mathrm{~g}$ for $2 \mathrm{~d}$ (before being ramped down to $100 \mathrm{~g}$ for testing).

The undrained shear strength $s_{\mathrm{u}}$ of the soil samples was determined using a $t$-bar penetrometer developed by Stewart and Randolph (1994). The strength profiles of the soil samples adopted in the $1 \mathrm{~g}$ and centrifuge test are shown in Figure 11. The magnitude of the undrained shear strength within the critical depth, which is within the footprint toe level $(z / D=$ $0 \cdot 33$ ), was comparable between the $1 \boldsymbol{g}$ and centrifuge samples.

\subsection{Creation of footprint and testing procedure}

In the $1 \boldsymbol{g}$ tests, a footprint was cut from the clay sample using a $v$-shaped blade that was centrally rotated to remove soil and to form a cavity of conical shape $20 \mathrm{~mm}$ deep $\left(z_{\mathrm{F}} / D=0 \cdot 33\right)$, with a radius of $60 \mathrm{~mm}(1 D)$. This caused minimal disturbance of the soil in the vicinity of the idealised footprint cavity, as reported by Kong (2012) and Kong et al. (2013).

A more realistic offshore footprint was created in-flight during the beam centrifuge tests. The footprint was created by penetrating the jack-up model spudcan vertically to a final depth $z_{\mathrm{FC}}=70 \mathrm{~mm}\left(z_{\mathrm{FC}} / D=1 \cdot 16\right)$ into the soil sample. The model was held at this depth for 10 min to simulate an operation time of $70 \mathrm{~d}$ at prototype scale. The physical jack-up model was then extracted from the soil, leaving a seabed depression surrounded by highly disturbed soil (and of geometric shape similar to the idealised cavity).

The full jack-up model prescribed by the real-time hybrid testing method was then reinstalled with a $1 \cdot 0 \mathrm{D}$ offset from the footprint's centre (see Figures 4 and 12 for definition). In the centrifuge, this was performed $20 \mathrm{~min}$ after complete extraction of the spudcan that made the footprint, simulating an elapsed time of $140 \mathrm{~d}$. The reinstallations were conducted to a 


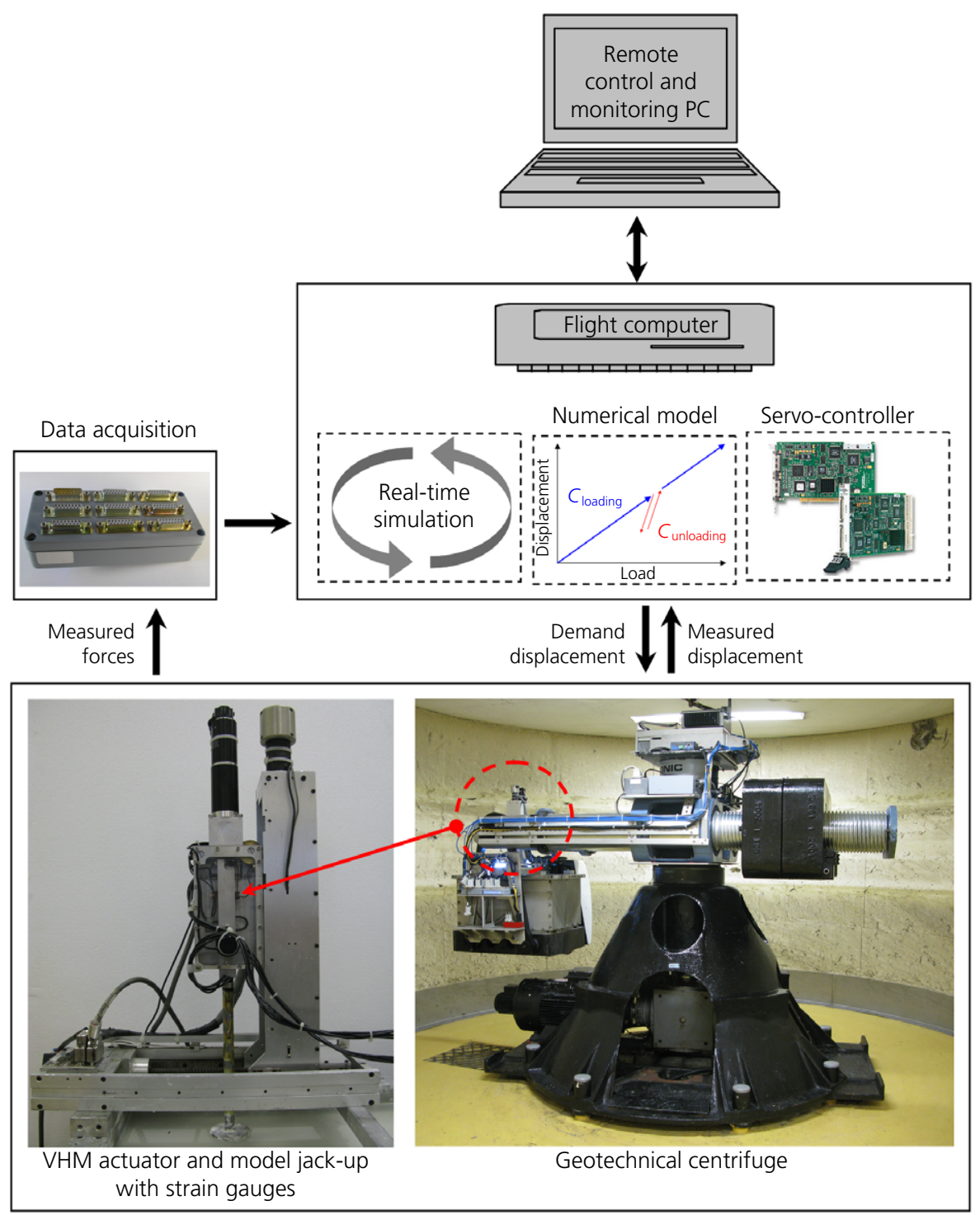

Figure 9. Outline of real-time hybrid testing with a geotechnical centrifuge for the modelling of jack-up reinstallation near existing footprints

maximum depth $z_{\max }$ of $100 \mathrm{~mm}\left(z_{\max } / D=1 \cdot 67\right)$, which was $0 \cdot 5 D$ below $z_{\mathrm{FC}}$. Further details regarding the effects of operation and elapsed time on the footprint's soil strength can be found in Gan (2009) and Gan et al. (2012).

\subsection{Example $1 g$ tests: effect of including horizontal flexibility}

The first phase of the $1 \boldsymbol{g}$ model tests considered the jack-up reinstallation response with a partially restrained connection at the model leg and actuator. The model jack-up had finite horizontal flexibility $C_{H h}$ and was allowed to slide according to the prescribed horizontal flexibility with the measured load.
This is equivalent to a horizontal spring connection between the model jack-up leg and actuator. The tests are labelled S-H-1 and S-H-2, with values of horizontal flexibility $C_{H h}$ provided in Table 4. Test S-R-1 was a rigid installation test. This refers to the reinstallation of the model jack-up leg rigidly connected to the actuator with no flexibility at the CP (similar to all tests previously performed by Cassidy et al., 2009; Gan, 2009; Kong et al., 2013; Stewart and Finnie, 2001).

Figure 13 shows the results of the three tests performed with different $C_{H h}$, S-H-1, S-H-2 and S-R-1, in terms of evolution of vertical and horizontal loads with penetration, horizontal 


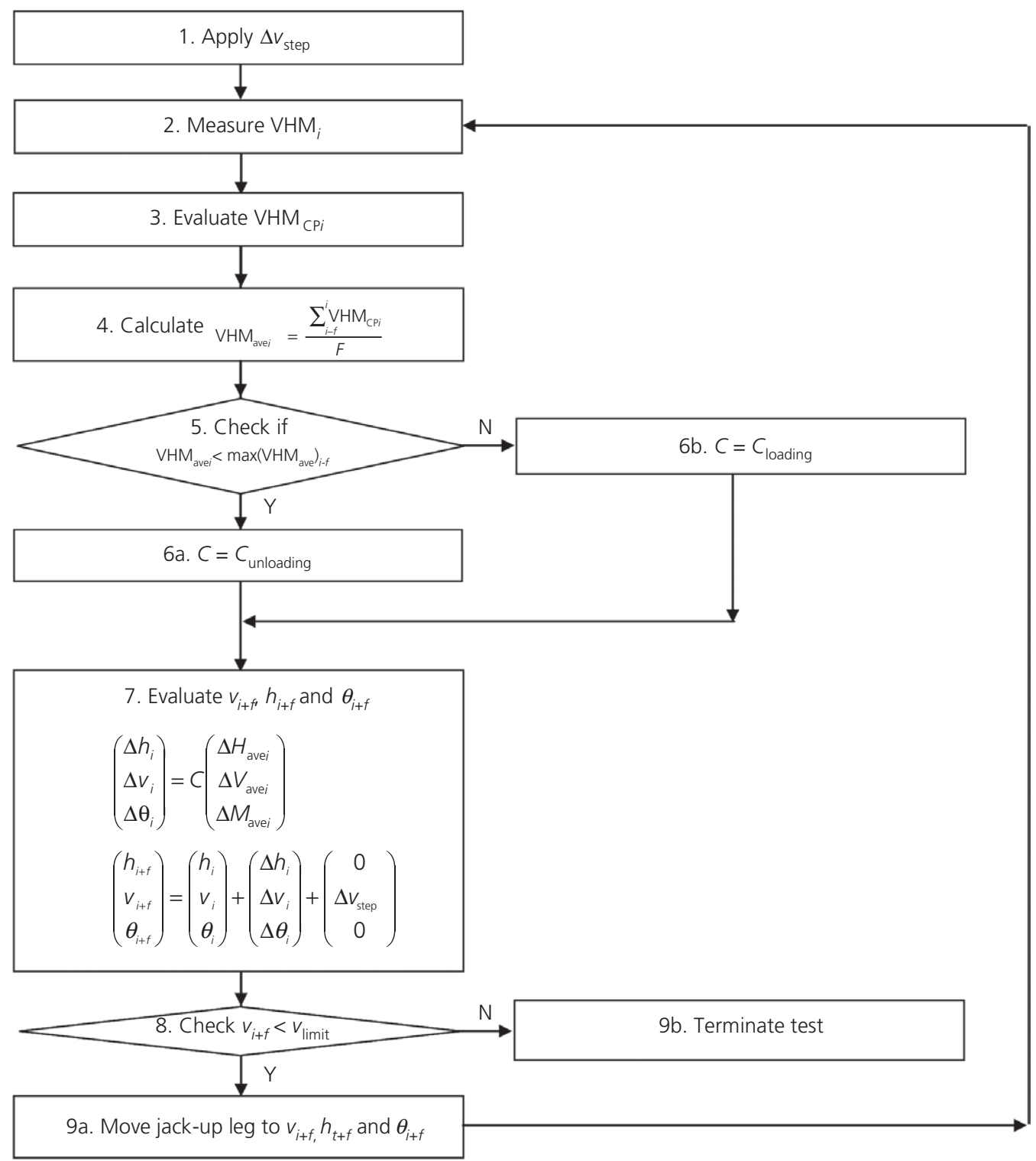

Figure 10. Flowchart of combined numerical model and real-time control algorithm

displacement with penetration, moments at the load reference point and at the $\mathrm{CP}$ with penetration and rotation of the leg with penetration.

When the footing first came into contact with the soil, the soil reaction pushed the footing towards the footprint centre, and, with the horizontal flexibility considered in tests S-H-1 and $\mathrm{S}-\mathrm{H}-2$, the footing started to move horizontally towards the footprint centre. It continued to slide towards the footprint centre until the bottom of the footprint $z_{\mathrm{F}}$ was passed. With this horizontal movement, the soil reaction was released, after which $H$ dropped significantly and became slightly negative.
The maximum horizontal displacements $\left(h_{\max }\right)$ increased from 0 to $3.3 \mathrm{~mm}\left(h_{\max } / D=0.055\right)$ to $9.5 \mathrm{~mm}\left(h_{\max } / D=0.158\right)$ as the horizontal flexibility increased between tests S-R-1, S-H-1 and $\mathrm{S}-\mathrm{H}-2$, respectively. The increase in $h_{\max }$ was proportional to the increase in horizontal flexibility (by a ratio of 3 ).

However, the tests confirmed that with horizontal movements there is a decrease in the horizontal loads (Figure 13). For instance, the maximum horizontal load on the footing $\left(H_{\max }\right)$ decreased by 22 and $14 \%$ in S-H-1 and S-H-2, respectively, compared with the rigid reinstallation test S-R-1 (peak values available in Table 5). The trends of change in horizontal load 


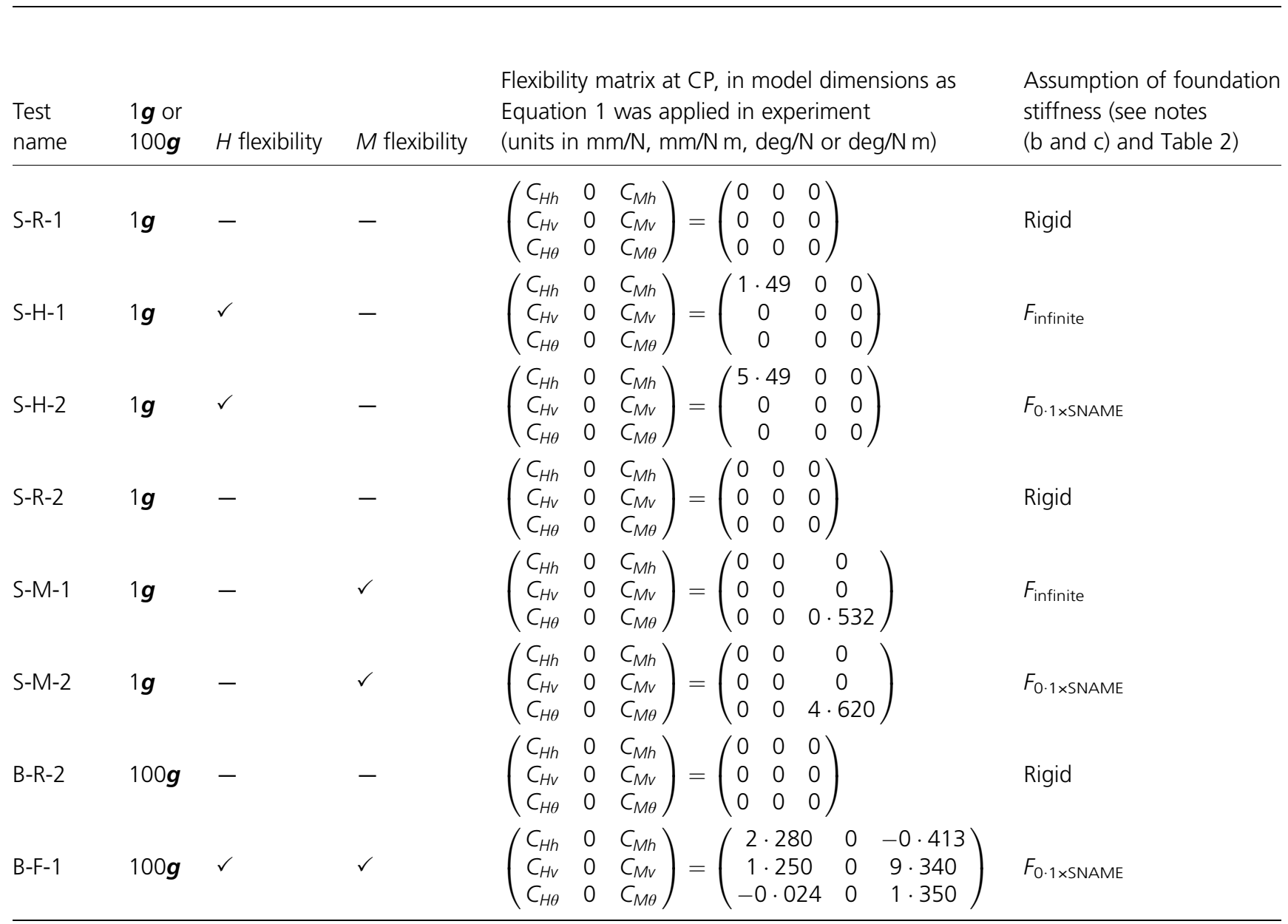

(a) The nomenclature of the tests is in the form of 'test type-flexibility matrix type-test number'. For the first letter, 'S' stands for $1 \boldsymbol{g}$-scaled model test and ' $\mathrm{B}$ ' for a beam centrifuge test at $100 \boldsymbol{g}$. For the different flexibility type adopted, the nomenclature is as follows: ' $\mathrm{R}$ ' for zero flexibility as the model jack-up leg was fully fixed to the actuator, ' $H$ ' for horizontal flexibility only, ' $M$ ' for rotational flexibility only and ' $F$ ' a full flexibility matrix. The test number refers to the number of similar tests conducted in series.

(b) A reinstallation test was conducted with the model leg fully fixed to the actuator connection and the model jack-up leg could only move vertically into the soil (tests S-R-1 and B-R-2). This is referred to as rigid reinstallation in subsequent discussions. This rigidity is different from the foundation rigidity defined by $F_{\text {infinite. }} F_{\text {infinite }}$ is an assumption of the numerical models having two pre-embedded footings rigidly connected to the ground. The reinstalling leg could still slide or rotate during reinstallation because the jack-up itself has finite flexibility.

(c) $\mathrm{F}_{0.1 \times \text { sname }}$ is an assumption of the numerical models having two footings with stiffness calculated at $10 \%$ of the stiffness matrix calculated with the SNAME guidelines, with the $100 \%$ values given in Table 2 .

(d) This table is a subset of the ten $1 \boldsymbol{g}$ tests and six $100 \boldsymbol{g}$ tests reported in Kong (2012).

Table 4. Flexibility matrix at the CP for the tests presented

with penetration depth in S-H-1 and $\mathrm{S}-\mathrm{H}-2$ were similar and exhibited only an $8 \%$ difference in $H_{\max }$ values. Up until the peak $M_{\mathrm{LRP}}$ value the moment was insensitive to changes in horizontal flexibility, with the $\left(M_{\mathrm{LRP}}\right)$ values obtained for the three tests only showing approximately $3 \%$ difference (Table 5).

Contrary to the rigid reinstallation case, the values of horizontal load in S-H-1 and SH-2 did not peak at $z / D=0 \cdot 2$ as penetration continued. Instead, the values peaked at approximately $z / D=0 \cdot 3$, after which they remained nearly constant until penetration went below $z / D=0 \cdot 6$. Rather than showing an abrupt peak in $M_{\mathrm{LRP}}$, as was the case for the rigid test, the moment at the footing took the penetration up to $z / D=0.6$ to reduce. The ability for the spudcan to displace horizontally seems to maintain the forces for a longer depth of penetration. 


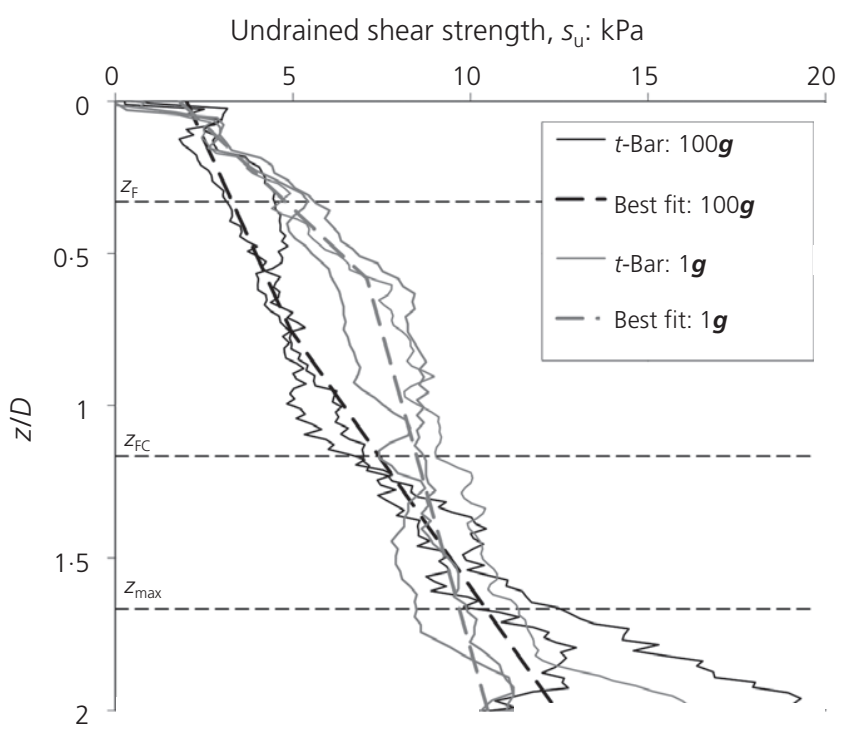

Figure 11. Undrained shear strength profiles of soil samples

\subsection{Example $1 g$ tests: effect of including rotational flexibility}

Three jack-up reinstallation tests were conducted at $1 \boldsymbol{g}$ to investigate the effect of including rotational flexibility in isolation. Finite rotational flexibility was prescribed so that the model leg could rotate about the $\mathrm{CP}$ of the jack-up model (see tests S-M-1 and S-M-2 in Table 4). This is equivalent to a hinge connection of finite stiffness. Figure 14 shows the effects of including rotational flexibility during reinstallation. In these tests, rotation at the $\mathrm{CP}$ also induced an equivalent horizontal displacement at the footing's LRP.

Initially during these reinstallation tests with rotational flexibility, the soil reaction simultaneously rotated and pushed the footing towards the footprint centre. At the $\mathrm{CP}$, the negative moment induced by the horizontal load was larger than the positive moment, the LRP. Thus, the moment at the $\mathrm{CP}$ was negative, and the footing rotated towards the footprint centre. The soil load was then released with the rotation. Both moments were therefore reduced. The horizontal load was also reduced as the footing rotated into the footprint. This was because there was equivalent horizontal displacement at the footing level as the jack-up leg rotated. Nonetheless, the release of the soil reaction was insufficient to keep the soil from bending the footing towards the footprint centre, and the $M_{\mathrm{CP}}$ remained negative.

As penetration continued, the trends in horizontal load and moment at the $\mathrm{CP}$ for tests S-M-1 and S-M-2 began to significantly differ. In test S-M-1, the footing rotated from

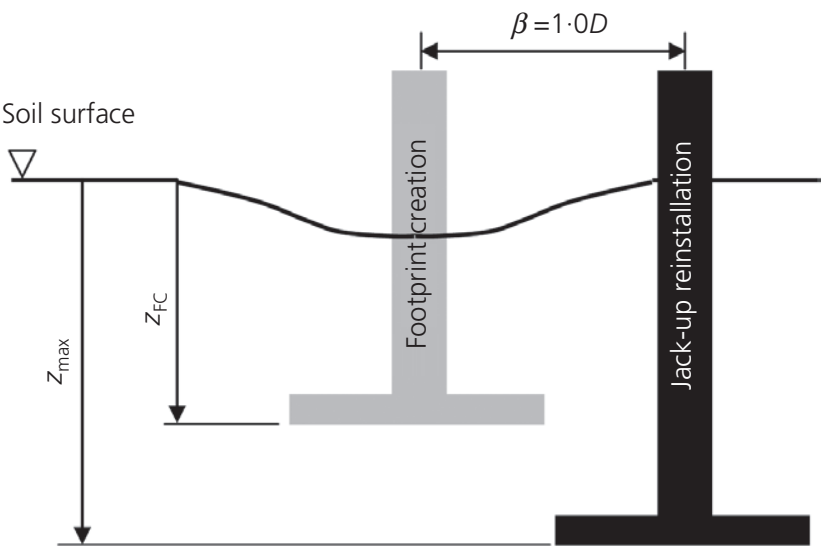

Figure 12. Schematic diagram of footprint creation and jack-up reinstallation with terminology definitions

touchdown level such that the angle of rotation increased in a linear manner until reaching maximum $M_{\mathrm{CP}}$ near the footprint toe level $(z / D \approx 0 \cdot 33)$. The moment at the $\mathrm{CP}$ decreased sharply with further penetration.

In summary, when rotational flexibility was included the footing rotated towards the footprint centre. The maximum angle of rotation $\left(\theta_{\max }\right)$ in S-M-1 and S-M-2 was $0 \cdot 275^{\circ}$ and $0.699^{\circ}$, respectively. $H_{\max }$ and $\left(M_{\mathrm{LRP}}\right)_{\max }$ were reduced by 40 and $20 \%$, when the rotational flexibility was increased from 0 to $C_{M \theta}=0.532^{\circ} / \mathrm{N} \mathrm{m}$, respectively (S-R-2 to S-M-1, see Table 5). The rotational flexibility of test S-M-2 $\left(C_{M \theta}=\right.$ $\left.4 \cdot 620^{\circ} / \mathrm{N} \mathrm{m}\right)$ was 8.5 times larger than tests S-M-1. $H_{\max }$ and $\left(M_{\mathrm{LRP}}\right)_{\max }$ were reduced by approximately 65 and $30 \%$, respectively, when compared with S-R-2 (Table 5). Unlike the horizontal flexibility tests, the load-displacement relationships were highly non-linear, and the $\theta_{\max }$ increased by 2.5 times with an 8.5 times increase in $C_{M \theta}$.

\subsection{Example beam centrifuge tests: effect of including horizontal and rotational flexibility}

The tests involved the reinstallation of the spudcan in an overconsolidated clay sample at a distance of $1.0 D$ to a pre-existing footprint, with two different stiffness matrices. The first test, B-R-2, assumes a fully rigid stiffness (i.e. $C=0$ ), whereas test B-F-1 assumes a stiffness matrix calculated from the SNAME (2002) guidelines (see Tables 3 and 4). While test B-R-2 is with full rigidity, test B-F-1 adopted a full flexibility matrix (vertical-horizontal-rotational cross-coupled) and the model jack-up was reinstalled at $1 \cdot 0 D$ from a real footprint. Therefore, all three governing parameters - that is, the footprint geometry, soil heterogeneity and structural properties of the jack-up unit were investigated in test B-F-1. 


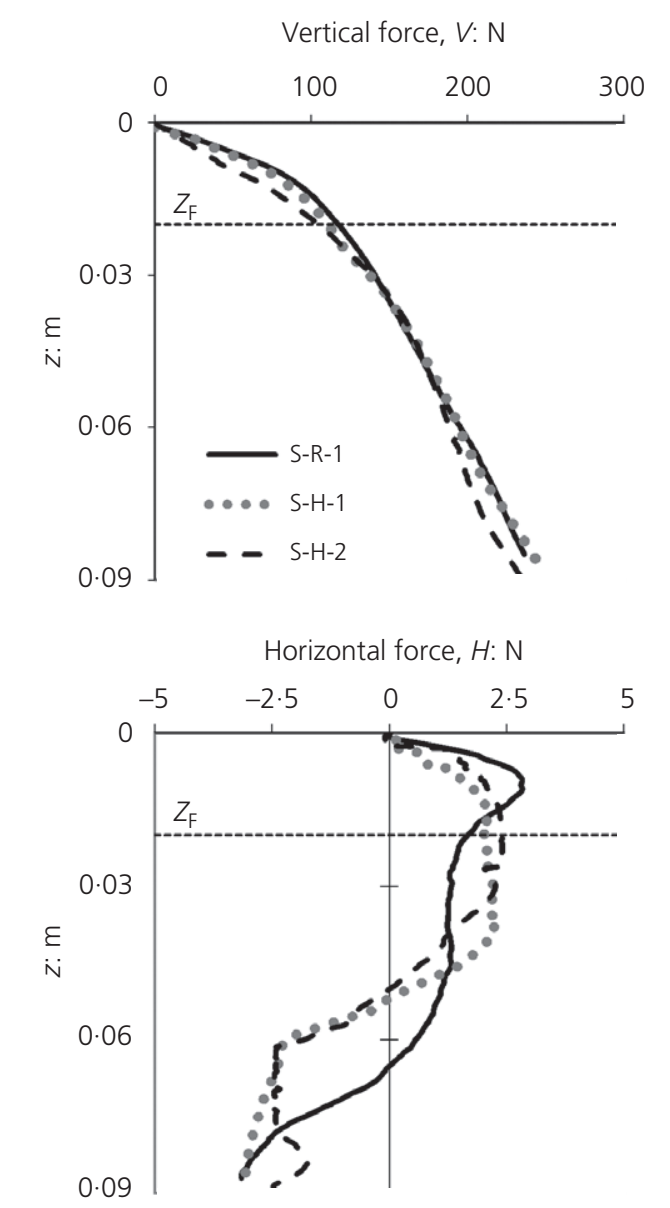

Moment at LRP, $M_{\mathrm{LRP}}: \mathrm{N} \mathrm{m}$

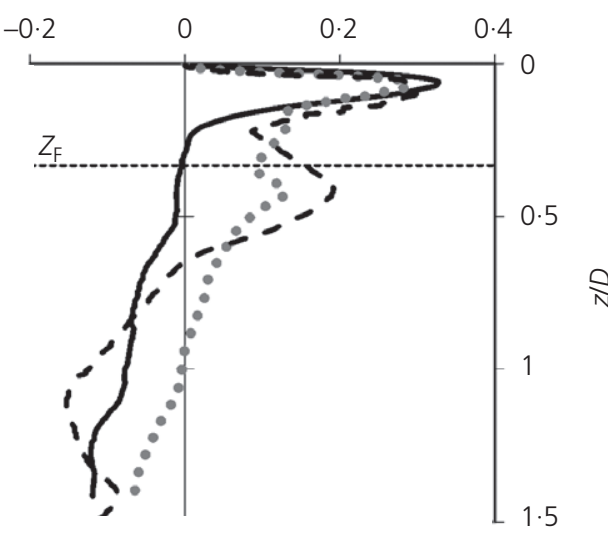

Moment at common point, $M_{C P}: \mathrm{N} \mathrm{m}$
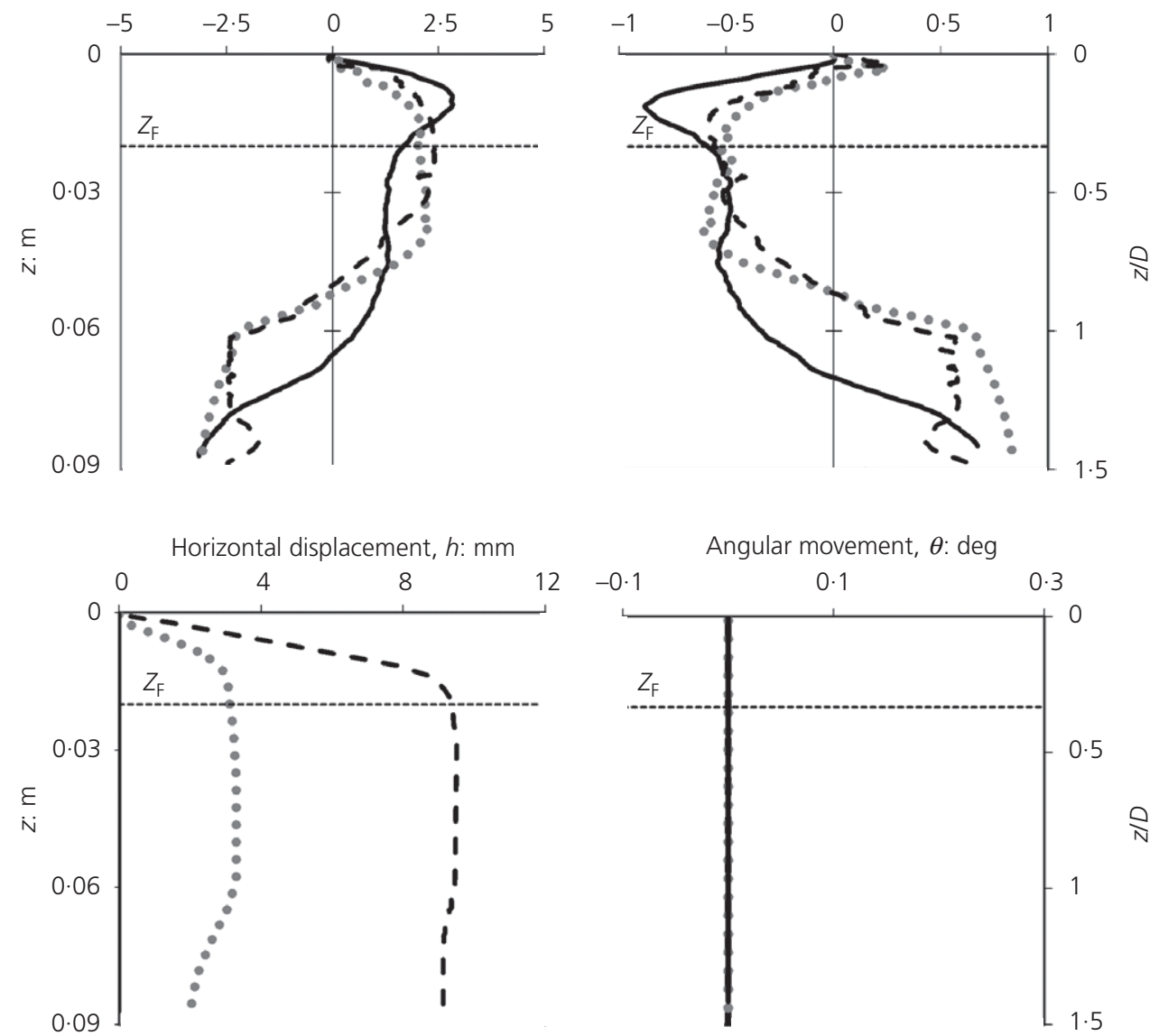

Figure 13. Reinstallation responses of $1 \boldsymbol{g}$ tests with different horizontal flexibility at $\mathrm{CP}$

Figure 15 shows the results of the two tests. The overall trend of development of loads and displacements with penetration is different between the two tests, highlighting the influence of the stiffness matrix and validating the performance of the system.
In test B-F-1, the jack-up leg initially exhibits a sharp increase in horizontal displacement, associated with an increase in horizontal load, as the spudcan is sliding into the footprint. The maximum horizontal displacement $h_{\max }$ during shallow depth 


\begin{tabular}{lcccccccc} 
Test & $H_{\text {max: }}: N$ & $H_{\text {max }} / A s_{u}$ & $M_{\text {lrp max: }}$ N m & $M_{\text {lrp max }} / A s_{u} D$ & $M_{\text {cp max:N m }}$ & $M_{\text {cp max }} / A s_{u} D$ & $h_{\text {max: }}$ mm & $\theta_{\text {max: }}$ deg \\
\hline S-R-1 & 2.84 & 0.34 & 0.33 & 1.03 & -0.78 & -1.57 & - & - \\
S-H-1 & 2.26 & 0.20 & 0.29 & 0.84 & -0.61 & -0.77 & 3.30 & - \\
S-H-2 & 2.42 & 0.23 & 0.30 & 0.84 & -0.58 & -0.95 & 9.51 & - \\
S-R-2 & 3.78 & 0.44 & 0.32 & 0.97 & -0.92 & -1.70 & - & - \\
S-M-1 & 2.25 & 0.23 & 0.26 & 0.73 & -0.61 & -0.89 & - & -0.28 \\
S-M-2 & 1.38 & 0.24 & 0.22 & 0.71 & -0.32 & -0.43 & - & -0.70
\end{tabular}

Table 5. Summary of peak responses at shallow depths for

$1 \boldsymbol{g}$ tests

reinstallation was $508 \mathrm{~mm}$ and the $\theta_{\max }$ was $0.8^{\circ}$. Once the penetration has reached the depth of the footprint, the horizontal load decreases significantly, resulting in a horizontal displacement away from the footprint. The moment at the load reference point shows a similar trend, associated with a change in angular rotation. As penetration continued into stronger soil (near $z_{\mathrm{FC}}$ ), the loading at footing level gradually increased and pushed the footing away from the footprint centre.

At touchdown level, the footing moved rapidly, sliding and rotating towards the footprint centre. The jack-up leg also rose up to $200 \mathrm{~mm}$ (prototype). This negative vertical movement was induced from the relatively large vertical-moment coupling flexibility term $\left(C_{M v}\right)$ (see Figure 8 and Table 5) and the negative $M_{\mathrm{CP}}$. As the footing rose up, the soil loading reduced rapidly and fluctuated around zero. As penetration continued, the loading at the footing level gradually increased. The vertical movement resulting from the $H$ and the $M_{\mathrm{CP}}$ cancelled each other, and the jack-up leg penetrated the soil at an almost constant rate. The footing started to move in the reverse direction (sliding and rotating away from the footprint centre) for penetration below the footprint toe level. At $z / D=$ $0 \cdot 8$, the strong soil layer near $z_{\mathrm{FC}}$ resulted in a relatively large negative $H$ and positive $M_{\mathrm{LRP}}$ and triggered movement in the vertical direction. The jack-up leg dropped down by $300 \mathrm{~mm}$ (prototype). As a result, the vertical, horizontal and moment loads increased rapidly. However, with further penetration, the horizontal force and moment gradually returned to their values before the reduction.

By allowing the jack-up leg to slide, rotate and rise/penetrate, the shallow depth reinstallation peak response was eliminated as the horizontal force and moment decreased to almost zero. The footing initially slid and rotated towards the footprint centre. The stronger soil near $z_{\mathrm{FC}}$ helped to push the footing back to its original position. Although the loading still peaks near $z_{\mathrm{FC}}$, the maximum horizontal load at depth and the moment at the CP were reduced by 43 and $16 \%$, respectively, compared with the rigid reinstallation conditions (peak values provided in Table 6).
In practice, if a jack-up could sustain the movement (and loading) during shallow depth reinstallation, with further penetration, the reverse loading at deep reinstallation could correct the movement back to almost its original position. Alternatively, a larger footing (or skirted footing) could be adopted to reduce the penetration depth requirement; this could avoid the large soil load near $z_{\mathrm{FC}}$.

The parallel between the evolution of horizontal loads and displacements and moment and rotation, as well as the concomitance of the inflexion point demonstrate the performance of the system and notably its reactivity to a change in loading conditions. The difference in response between these tests demonstrates that the real-time hybrid system is capable of performing even for a complex load-displacement input and at the high gravity level in the beam centrifuge.

\section{Conclusions}

The real-time hybrid testing method pioneered in structural dynamic testing has been extended to a geotechnical application in a centrifuge. This paper provides a detailed description of the application of this powerful and novel technique in modelling the jack-up reinstallation problem.

Although they are known to be governing parameters, the effects of the structural properties of a full jack-up unit on the unit's reinstallation responses had never previously been investigated due to testing equipment limitations and the assumption that the jack-up unit was fully rigid at the leg-hull connection level, ignoring the finite structural flexibility of the unit. Therefore, this study was the first to investigate the reinstallation response of a full jack-up unit. Simplification of the jack-up was still required with the numerical component of the hybrid method assuming a static and elastic structure (and spudcans-soil interaction). However, the real-time hybrid method outlined can accommodate a changing flexibility matrix at the $\mathrm{CP}$, and therefore, future studies could use a nonlinear and dynamics numerical model to allow a more realistic prototype scaling to be tested. 

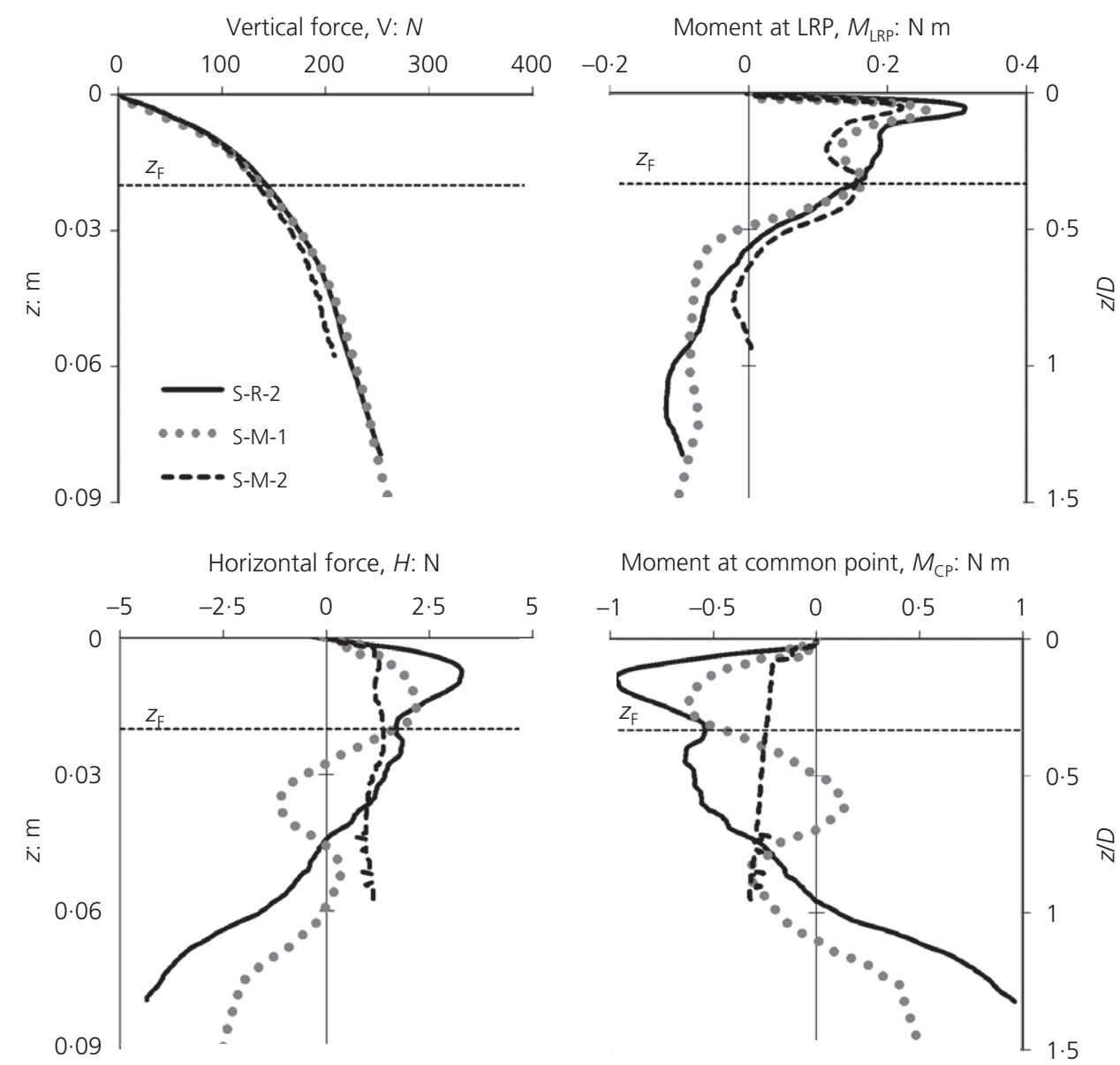

Moment at common point, $M_{\mathrm{CP}}: \mathrm{N} \mathrm{m}$
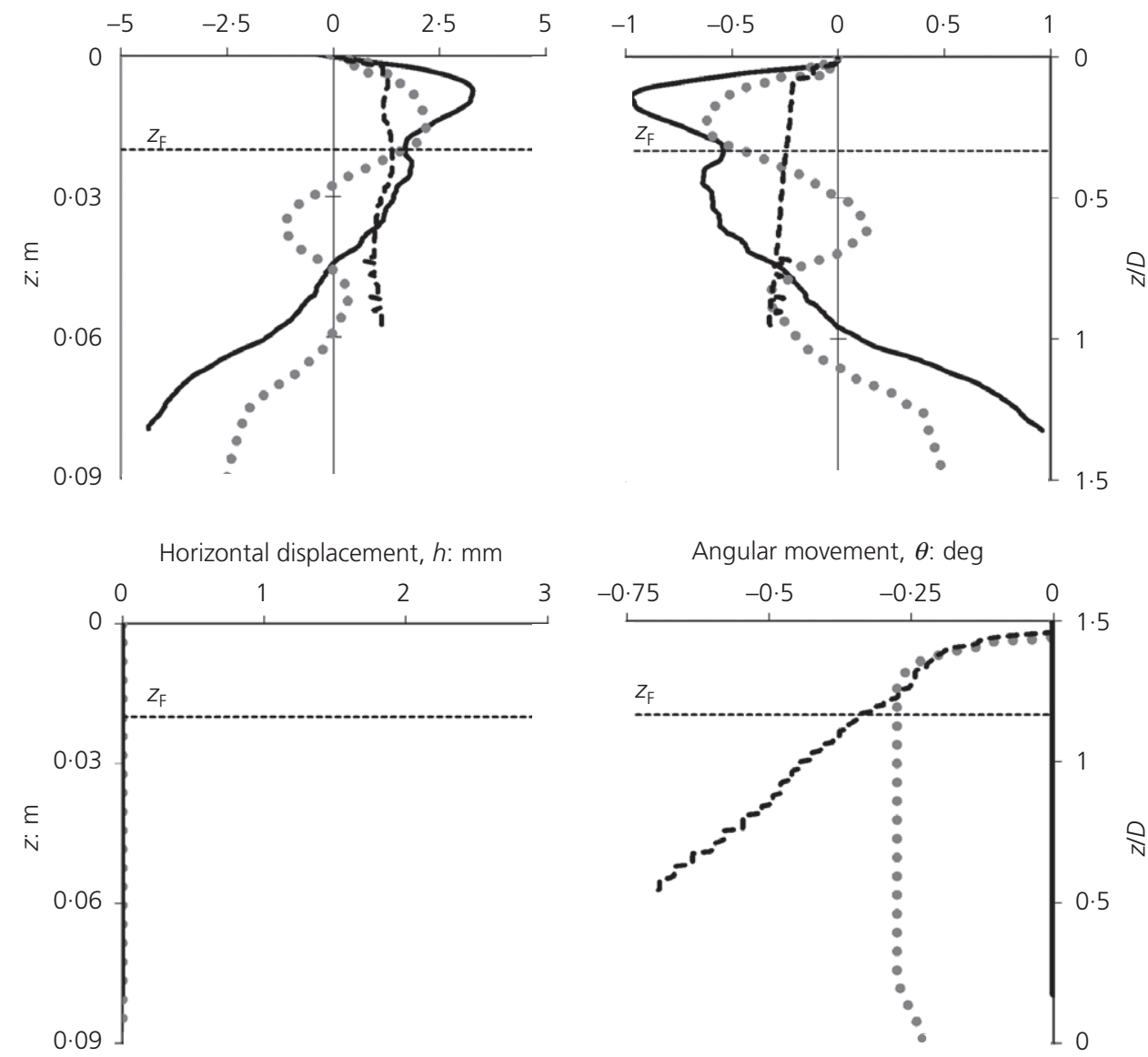

Angular movement, $\theta$ : deg

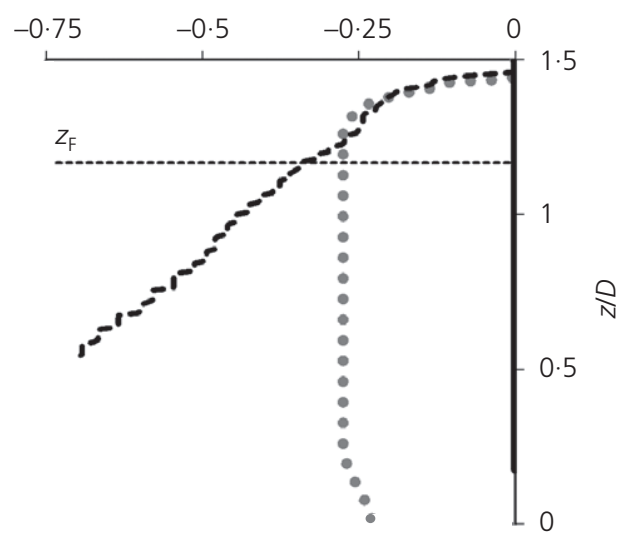

Figure 14. Reinstallation responses of $\mathbf{1 g}$ tests with different rotational flexibility at $\mathrm{CP}$ 


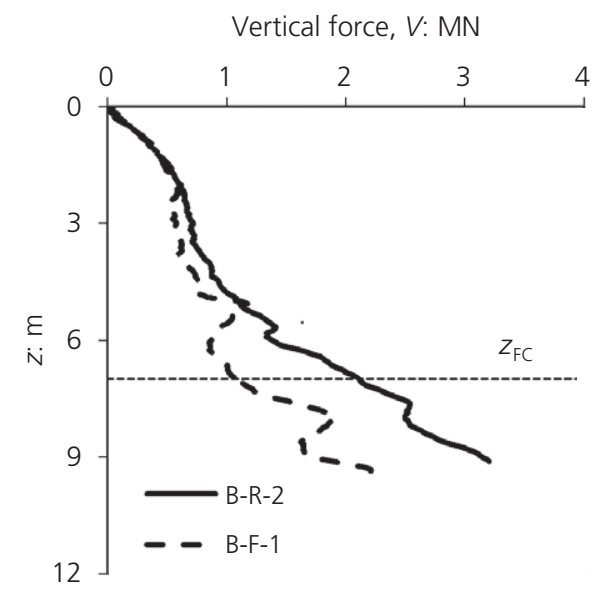

Moment at LRP, $M_{\text {LRP }}: M N$ m

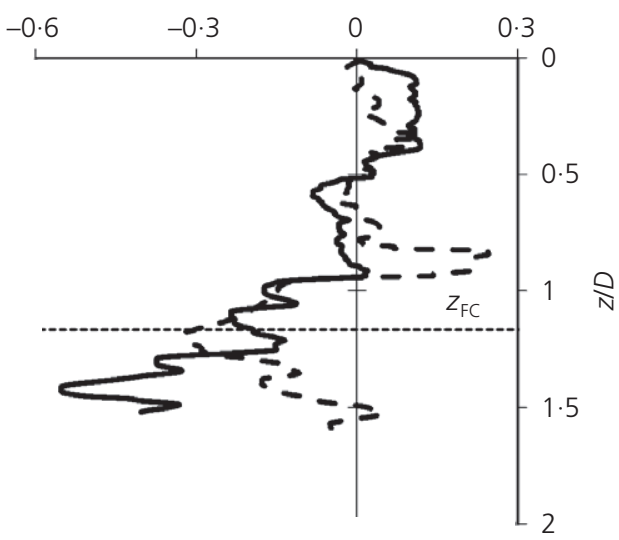

Horizontal force, $H$ : MN

Moment at common point, $M_{\mathrm{CP}}: \mathrm{MN} \mathrm{m}$
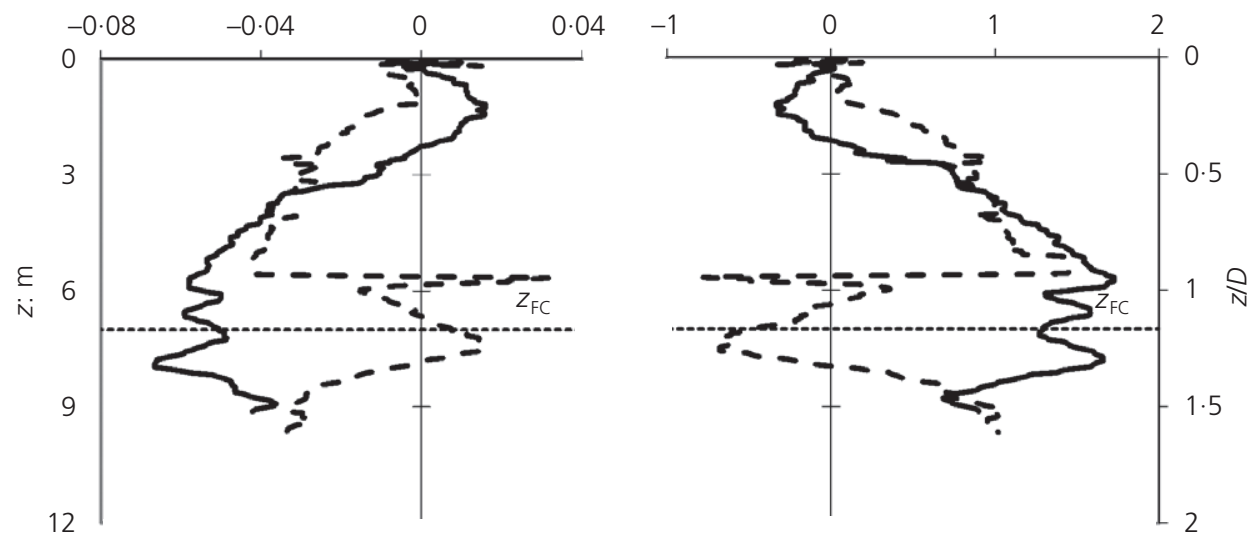

Prototype horizontal displacement, $h: \mathrm{mm}$

Angular movement, $\theta$ : deg
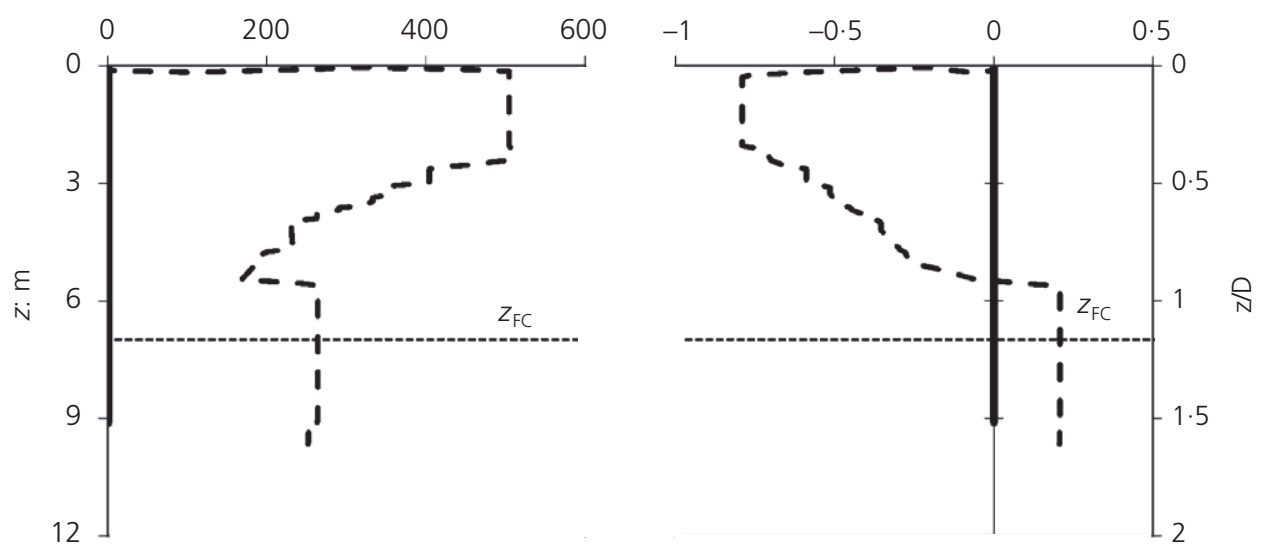

Figure 15. Reinstallation response from beam centrifuge tests 


\begin{tabular}{|c|c|c|c|c|c|c|c|c|}
\hline Test & $H_{\text {max }}: M N$ & $H_{\text {max }} / A s_{\mathrm{u}}$ & $M_{\text {Irp max }}: M N$ m & $M_{\text {Irp max }} / A s_{\mathrm{u}} D$ & $M_{\mathrm{cp} \text { max }}: \mathrm{MN} \mathrm{m}$ & $M_{\mathrm{cp} \max } / A s_{\mathrm{u}} D$ & $h_{\max }: \mathrm{mm}$ & $\theta_{\text {max }}:$ deg \\
\hline B-R-2 & -0.07 & -0.36 & -0.55 & -0.24 & 1.69 & $1 \cdot 78$ & - & - \\
\hline B-F-1 & -0.04 & -0.23 & -0.29 & -0.20 & $1 \cdot 42$ & $1 \cdot 50$ & $251 \cdot 3$ & -0.21 \\
\hline
\end{tabular}

Table 6. Summary of peak responses at deep depths for beam centrifuge tests

By using the real-time hybrid testing method in a beam centrifuge, all three key parameters in the jack-up reinstallation problem - the footprint geometry, soil heterogeneity and structural properties of the jack-up unit - were included, forming a complete model. The footing initially slid and rotated towards the footprint centre, but the stronger soil below the bottom of the footprint cavity helped to push the footing back to its original position. Major, significant differences between the results of using the hybrid apparatus and results of using less adaptive boundary constraints (a fixed system) are shown in this paper. This more realistic testing of complex non-linear conditions is the major advantage of the real-time hybrid testing method, and for the jack-up reinstallation problem the results are considerably more informative, accurate and useful.

\section{Acknowledgements}

The lead author acknowledges the support of an Australian postgraduate scholarship. The authors also thank Mr Phil Hortin and Mr Shane De Catania for their contribution to the development of the experimental apparatus and software. The work forms part of the activities of the Centre for Offshore Foundation Systems (COFS), which is supported by the Lloyd's Register Foundation and is now forming one of the primary nodes of the Australian Research Council (ARC) Centre of Excellence in Geotechnical Science and Engineering. The authors thank the anonymous reviewers for their comments and advice that improved the final manuscript.

\section{Appendix \\ A1 Detailed design, fabrication and calibration of the VHM actuator}

This appendix provides details of the design, fabrication and calibration of the new VHM actuator. Further details are available in Kong (2012).

\section{A2 Detailed description of the motors and cable}

A DC servo motor module composed of a Maxon RE-40-150W Graphite Brushes Motor, a 546:1 Maxon GP42C Planetary GearHead gearbox and a 2000 count Maxon HEDS $5540500 \mathrm{CPT} 3$ channels encoder was selected to fulfil the speed, torque and precision requirements. The selected motor module with a precision of $1.49 \times 10^{-5} \%$ count which is in the same order with the requirement was considered to be sufficient. The precision requirement was the critical motor selection criteria.

The pulley cable was designed to withstand the design working load with a minimum factor of safety of $1 \cdot 5$, hence $225 \mathrm{~N}$. The $1.0 \mathrm{~mm}$ diameter strained stainless steel wire with a maximum tensile capacity of $1700 \mathrm{~N}$ was selected. This was significantly higher than the design requirement. This is because the cable was selected for its flexibility to form a tight loop around the pulleys, rather than based on strength. The cable was pretensioned to $300 \mathrm{~N}$ before use, so as to reduce the potential slack in the pulley system.

This reduced the tensile capacity to $1400 \mathrm{~N}$. With such a tight pulley system, the rotational movement at the motor output shaft could be accurately transferred to angular movement at the pivot point. To monitor and to adjust the pre-tension level in the cable, the cable was connected to a strain gauge at each end. The cable was firmly connected to the arm through a nutscrew connection at the end of the cable. The arm was designed to rotate around the pivot point. The pivot point comprised of a series of miniature ball bearings and needle bearings to provide smooth rotational motion under the design load. To prevent out-of-range movement, two limit switches were installed on the inner wall of the actuator box. If tripped, the motor immediately stops to ensure no damage to pulley systems. The output shaft of the motor module was attached to an actuator box that contains all the other components. The actuator box was designed with a transition piece allowing the actuator to be rigidly bolted to the tower of the existing linear actuator unit.

\section{A3 Sources of error and calibration}

Like any motor system, the selected motor module is subjected to backlash when the motor reverses the direction of driving. On the basis of the manufacturer's specification, the theoretical backlash at the pivot point is $0 \cdot 026^{\circ}$. This is considered to be insignificant compared with the design angular stroke.

The stainless steel cable also extends when subjected to tension load. The theoretical extension of a $290 \mathrm{~mm}$ long cable under working load $(141 \mathrm{~N})$ is $0.1 \mathrm{~mm}$. This corresponds to an angular movement of $0.06^{\circ}$ at the pivot point. Again, this error is approximately $3 \%$ of the maximum angular movement and was considered to be acceptable. 
Development of a real-time hybrid testing method in a centrifuge

Kong, Cassidy and Gaudin
Angular reading

\begin{tabular}{lccc}
$\begin{array}{l}\text { from motor } \\
\text { driving: deg }\end{array}$ & $\begin{array}{c}\text { Front encoder } \\
\text { reading: deg }\end{array}$ & $\begin{array}{c}\text { Difference : } \\
\text { deg }\end{array}$ & $\begin{array}{c}\text { Difference: } \\
\%\end{array}$ \\
\hline+3 & +3.020 & 0.020 & 0.67 \\
-3 & -2.995 & 0.005 & 0.17
\end{tabular}

Table A1. Calibration of angular reading from front encoder

To assess accurately the actual angular movement at the pivot point, an additional encoder (named the front encoder) was mounted on top of the pivot point. This front encoder is free from the backlash and cable extension error. The front encoder (80000 count Avago AED-3300-BE1 encoder) had a precision of $4.50 \%$ count, which was the highest available in the market at the time of design. However, the precision is still substantially less than the design precision requirement $\left(1 \cdot 4 \cdot 10^{5} \%\right.$ count $)$. Therefore, the angular movement was still prescribed through the motor module with higher precision and the front encoder was only used for validation of the magnitude of error and for monitoring purposes.

The new actuator was calibrated for accuracy in prescribed angular movement. The vertical position, that of zero rotation $(\theta=0)$, was first determined. Slip gauges, which are stainless steel blocks with precisely known thickness, were used for this. The actuator was driven until the gap between the arm and the side of the actuator box was exactly the same at the two ends of the arm. At that position, the arm is in the exact vertical position and zero rotation at the pivot point can be assumed.

Following this, the accuracy of the rotation of the leg was assessed by physical measurement and by direct comparison with the front encoder reading. The maximum anticipated angular movement of approximately $2^{\circ}$ was considered. The angular actuator was first driven from the absolute zero position to $1.9988^{\circ}$; the sine bar, parallel bar and slip gauges were used to determine the height at the two ends of the arm. The physical angular reading was then determined using basic trigonometry. The difference between the angular reading from the motor encoder and the physically measured angle was less than $0.01^{\circ}$, or $0.6 \%$ of that recorded. The accuracy of the angular movement was subsequently assessed by evaluating the different readings from motor driving and the front encoder. The front encoder provides accurate angular reading as it is free from backlash and cable extension error.

To access the variation of accuracy over the full range of angular movement, the actuator was first driven from $0^{\circ}$ to the maximum positive stroke of $3^{\circ}$. The actuator was then reversed and moved back to the zero position. Both the motor driving and the front encoder reading were reset to zero to eliminate the effect of backlash. Finally, the actuator was driven from $0^{\circ}$ to the maximum negative stroke of $-3^{\circ}$. A slight difference between the motor driving and the front encoder readings was recorded and it increased with angle. However, as detailed in Table A1, the maximum difference is within $0.67 \%$. The reading from the two encoders is therefore in excellent agreement.

The effect of backlash was assessed by driving and reversing the actuator in $0 \cdot 25^{\circ}$ increments, over the full range of angular stroke $\left( \pm 3^{\circ}\right)$. The maximum difference during the reverse of angular movement direction was recorded as $0.025^{\circ}$, which is similar to the manufacture's specification of $0.026^{\circ}$.

\section{REFERENCES}

Adachi T, Yamada M, Sekihara H and Enami A (1998)

Pseudo-dynamic test on intensity of earthquake ground motions at occurrence of liquefaction of subsurface layers. Journal of Structure Construction Engineering 505: 85-91 (in Japanese).

Ayari L (2008) Hybrid testing \& simulation - the next step in verification of mechanical requirements in the aerospace industry. In Hybrid Simulation - Theory, Implementation and Applications (Saouma V and Sivaselvan M (eds)). Taylor \& Francis, London, UK.

Bienen B, Cassidy MJ, Byron-Brown J and Byrne BW (2006) Three-dimensional modelling of offshore jack-up structures: An experimentally based research strategy. In Proceedings of the 6th International Conference on Physical Modelling in Geotechnics Hong Kong (Ng, Zhang and Wang (eds.)), Taylor \& Francis, Hong Kong, vol. 1, pp. 617-623.

Bienen B, Cassidy MJ and Gaudin C (2009) Physical modelling of the push-over capacity of a jack-up structure on sand in a geotechnical centrifuge. Canadian Geotechnical Journal 46(2): 190-207, http://dx.doi.org/10.1139/T08-114.

Blakeborough A, Williams MS, Darby AP and Williams DM (2001) The development of real-time substructure testing. Philosophical Transactions of the Royal Society of London. Series A 359: 1869-1991, http://dx.doi.org/10.1098/rsta. 2001.0877.

Cassidy MJ, Martin CM and Houlsby GT (2004) Development and application of force resultant models describing jack-up foundation behaviour. Marine Structures 17(3-4): 165-193, http://dx.doi.org/10.1016/j.marstruc.2004.08.002.

Cassidy MJ, Quah CK and Foo KS (2009) Experimental investigation of the reinstallation of spudcan footings close to existing footprints. Journal of Geotechnical and Geoenvironmental Engineering, ASCE 135(4): 474 486, http://dx.doi.org/10.1061/(ASCE)1090-0241(2009)135:4 (474). 
International Journal of Physical Modelling in Geotechnics Volume 15 Issue 4
Development of a real-time hybrid testing method in a centrifuge

Kong, Cassidy and Gaudin
Cassidy MJ, Vlahos G and Hodder MS (2010) Assessing appropriate stiffness levels for spudcan foundations on dense sand. Marine Structures 23(2): 187-208, http://dx. doi.org/10.1016/j.marstruc.2010.03.003.

Dean ETR, James RG and Schofield AN (1996) Drum Centrifuge Study of Three-Leg Jackup Models on Clay. Cambridge University Engineering Department, Cambridge, UK, Technical Report CUED/D-SoiWTR289.

Dean ETR, James RG, Schofield AN and Tsukamoto Y (1997) Theoretical modelling of spudcan behaviour under combined load. Soils and Foundations 37(2): 1-15, http://dx.doi.org/10.3208/sandf.37.2_1.

De Catania S, Breen J, Gaudin C and White DJ (2010) Development of a multiple axis actuator control system. Proceedings of the 7th International Conference on Physical Modelling in Geotechnics, Zurich, Switzerland. CRC Press, Zurich, Switzerland, vol. 1, pp. 325-330.

Fujii T, Hyodo M, Kusakabe S and Fukuda K (2000) On-line seismic response tests on embankments founded on saturated sandy deposits. Japanese Society of Civil Engineering (652): 229-243 (in Japanese).

Gan CT (2009) Centrifuge Model Study on Spudcan-Footprint Interaction. $\mathrm{PhD}$ thesis, National University of Singapore, Singapore.

Gan CT, Leung CF, Cassidy MJ, Gaudin C and Chow YK (2012) Effect of time on spudcan-footprint interaction in clay. Géotechnique 62(5): 401-413, http://dx.doi.org/10.1680/ geot.10.P.063.

Gaudin C, Cassidy MJ and Donovan T (2007) Spudcan reinstallation near existing footprints. Proceedings of the 6th International Offshore Site Investigation and Geotechnics Conference: Confronting New Challenges and Sharing Knowledge, London, UK. Society of Underwater Technology, London, UK, pp. 285-292.

Gaudin C, White DJ, Boylan N et al. (2009) A wireless data acquisition system for centrifuge model testing. Measurement Science and Technology 20: 095709, http://dx. doi.org/10.1088/0957-0233/20/9/095709.

Goodwin GC, Graebe SF and Salgado ME (2000) Control system design. Prentice Hall, Upper Saddle River, NJ, USA.

Hakuno M, Shidawara M and Hara T (1969) Dynamic destructive test of a cantilever beam, controlled by an analog-computer. Transactions of the Japan Society of Civil Engineers (171): 1-9, http://dx.doi.org/10.2208/ jscej1969.1969.171_1. (in Japanese).

Integrated Technical Software Pty Ltd. (2010) SPACE GASS Structural Engineering Design and Analysis Software (Release 10·81). Integrated Technical Software Pty Ltd, Geelong, Australia.

Katada T and Hakuno M (1982) On-line experimental analysis of surface ground motion in liquefaction process. 3rd International Earthquake Microzonation Conference, Seattle, WA, USA.
Kazama M, Sento N, Kwon Y and Yamaguchi A (2004) Geotechnical hybrid simulation of liquefiable inclined ground considering pore water migration. 13th World Conference on Earthquake Engineering, Vancouver, Canada, Paper No. 3475.

Kobayashi H, Tamura K and Tanimoto S (2002) Hybrid vibration experiments with a bridge foundation system model. Soil Dynamics and Earthquake Engineering 22(9-12): 1135-1141, http://dx.doi.org/10.1016/S0267-7261(02) 00140-9.

Kong VW (2012) Jack-Up Reinstallation Near Existing Footprints. PhD thesis, University of Western Australia, Perth, Australia.

Kong VW, Cassidy MJ and Gaudin C (2013) Experimental study of the effect of geometry on the reinstallation of a jack-up next to a footprint. Canadian Geotechnical Journal 50(5): 557-573, http://dx.doi.org/10.1139/cgj-2012-0381.

Kusakabe S and Morio S (1995) The development of a substructure on-line testing system for seismic response analysis of a geotechnical system. Soils and Foundations 35(2): 117-125, http://dx.doi.org/10.3208/sandf1972.35. 2_117.

Kwon Y, Kazama M and Uzuoka R (2007) Geotechnical hybrid simulation system for one-dimensional consolidation analysis. Soils and Foundations 47(6): 1133-1140, http://dx. doi.org/10.3208/sandf.47.1133.

Menzies D and Roper R (2008) Comparison of jackup rig spudcan penetration methods in clay. Offshore Technology Conference, Houston, TX, US.

Mochizuki T (1975) On-line real time dynamic test for building supported on piles, part 1 about the test method and restoring force characteristics of pile in cohesive soil. Transactions of Architectural Institution of Japan 231: 63-71 (in Japanese).

Murff JD, Hamilton JM, Dean ETR et al. (1991) Centrifuge testing of foundation behaviour using full jackup rig models. Offshore Technology Conference, Houston, TX, USA.

Nakashima M, Kato H and Takaoka E (1992) Development of real-time pseudo dynamic testing. Earthquake Engineering and Structural Dynamics 21: 779-92, http://dx.doi.org/10. 1002/eqe.4290210106.

National Instruments (2009) LabVIEW PID and Fuzzy Logic Tookit User Manual. National Instruments, Austin, TX, USA.

Ohtomo K, Sakai M, Dozono Y and Fukuyama M (2008) Hybrid simulation at CRIEPI: applications to soil structure interaction. In Hybrid Simulation - Theory, Implementation and Applications (Saouma V and Sivaselvan M (eds)). Taylor \& Francis, London, UK.

Punrattansin P, Nishioka H, Murata O and Kusakabe O (2003) Development of combined loading apparatus for centrifuge test. International Journal of Physical Modelling in 
International Journal of Physical Modelling in Geotechnics Volume 15 Issue 4
Development of a real-time hybrid testing method in a centrifuge

Kong, Cassidy and Gaudin
Geotechnics 3(4): 1-13, http://dx.doi.org/10.1680/ijpmg. 2003.3.4.01.

Sento N, Kazama M, Fukumoto S and Saito K (2002)

Geotechnical hybrid simulation of ground evaluation to be liquefiable by current conventional design method. US-Japan Seminar on Seismic Disaster Mitigation in Urban Area by Geotechnical Engineering, Anchorage, AK, USA.

SNAME (Society of Naval Architects and Marine Engineers) (2002) Technical \& Research Bulletin 5-5A. Guidelines for Site Specific Assessment of Mobile Jack-up Units. SNAME, Alexandria, VA, USA.

Spencer B, Elnashai A, Kuchma D et al. (2006) Multi-Site Soil-Structure-Foundation Interaction Test (MISST). University of Illinois at Urbana-Champaign, Champaign, IL, USA; Lehigh University, Bethlehem, PA, USA; and Rensselaer Polytechnic Institute, Troy, NY, USA.

Stewart DP (1992) Lateral Loading of Piled Bridge Abutments Due to Embankment Construction. PhD thesis, University of Western Australia, Perth, Australia.

Stewart DP and Randolph MF (1994) T-Bar penetration testing in soft clay. Journal of Geotechnical Engineering, American Society of Civil Engineers. 120(12): 2230-2235.

Stewart DP and Finnie IMS (2001) Spudcan-footprint interaction during jack-up workovers. 11th International Offshore and Polar Engineering Conference, Stavanger, Norway, pp. 61-65.

Takahashi N, Hyodo M, Yoshihiro $Y$, Kimura T and Yamamoto $Y$ (2003) On line pseudo-dynamic response test on stratified ground including clay layer. 3rd International Offshore and Polar Engineering Conference, Honolulu, HI, USA, pp. 466-473.

Van der Auweraer $\mathrm{H}$, Vecchio $\mathrm{A}$, Peeters $\mathrm{B}$, Dom $\mathrm{S}$ and Mas $\mathrm{P}$ (2008) Hybrid testing in aerospace and ground vehicle development. In Hybrid Simulation - Theory, Implementation and Applications (Saouma $\mathrm{V}$ and Sivaselvan M (eds)). Taylor and Francis, London, UK.

Vlahos G (2004) Physical and Numerical Modelling of A Three-Legged Jack-up Structure on Clay Soil. PhD thesis, University of Western Australia, Perth, Australia.

Vlahos G, Martin CM, Prior MS and Cassidy MJ (2005) Development of a model jack-up unit for the study of soil-structure interaction on clay. International Journal of Physical Modelling in Geotechnics 5(2): 31-48, http://dx. doi.org/10.1680/ijpmg.2005.5.2.31.

Vlahos G, Cassidy MJ and Martin CM (2008) Experimental investigation of the system behaviour of a model three-legged jack-up on clay. Applied Ocean Research 30(4): 323-337, http://dx.doi.org/10.1016/j.apor.2009.02.004.

Vlahos G, Cassidy MJ and Martin CM (2011) Numerical simulation of pushover tests on a model jack-up platform on clay. Géotechnique 61(11): 947-960, http://dx.doi.org/10. 1680/geot.8.P.114
Wagg D, Neild S and Gawthrop P (2008) Real-time testing with dynamic substructuring. In Modern Testing Techniques for Structural Systems - Dynamics and Control (Bursi OS and Wagg D (eds)). Springer, New York, NY, USA.

Wang Q, Wang J, Chi F, Jin F and Zhang C (2009) Real-time dynamic hybrid testing for soil-structure interaction. $3 \mathrm{rd}$ International Conference on Advances in Experimental Structural Engineering, San Francisco, CA, USA.

Zhang Y, Bienen B, Cassidy MJ and Gourvenec S (2011) The undrained bearing capacity of a spudcan foundation under combined loading in soft clay. Marine Structures 24(4): 459-477, http://dx.doi.org/10.1016/j.marstruc.2011.06.002.

Zhang Y, Bienen B and Cassidy MJ (2013) Development of a combined $V H M$ loading apparatus for a geotechnical drum centrifuge. International Journal of Physical Modelling in Geotechnics 13(1): 13-30, http://dx.doi.org/10. 1680/ijpmg.12.00007.

Zhang Y, Cassidy MJ and Bienen B (2014a) A plasticity model for spudcans in soft clay. Canadian Geotechnical Journal 51(6): 629-646, http://dx.doi.org/10.1139/cgj-2013-0269.

Zhang Y, Cassidy MJ and Bienen B (2014b) Jack-up push-over analyses featuring a new force resultant model for spudcans in soft clay. Ocean Engineering 81: 139-149, http://dx.doi.org/10.1016/j.oceaneng.2014.02.008.

Zhang Y, Wang D, Cassidy MJ and Bienen B (2014c) Effect of installation on the bearing capacity of a spudcan under combined loading in soft clay. Journal of Geotechnical and Geoenvironmental Engineering, American Society of Civil Engineers 140(7), http://dx.doi.org/10.1061/(ASCE)GT. 1943-5606.0001126.

\section{WHAT DO YOU THINK?}

To discuss this paper, please email up to 500 words to the editor at journals@ice.org.uk. Your contribution will be forwarded to the author(s) for a reply and, if considered appropriate by the editorial panel, will be published as discussion in a future issue of the journal.

International Journal of Physical Modelling in Geotechnics relies entirely on contributions sent in by civil engineering professionals, academics and students. Papers should be 2000-5000 words long (briefing papers should be 10002000 words long), with adequate illustrations and references. You can submit your paper online via www.ice virtuallibrary.com/content/journals, where you will also find detailed author guidelines. 